\title{
Hexosamine pathway activation improves memory but does not extend lifespan
}

in mice.

Running title: Hexosamine pathway activation in mammalian aging

Kira Allmeroth ${ }^{1 \#}$, Matías D. Hartman ${ }^{1 \#}$, Martin Purrio ${ }^{1}$, Andrea Mesaros ${ }^{1}$, Martin S.

Denzel ${ }^{1,2,3 *}$

${ }^{1}$ Max Planck Institute for Biology of Ageing

D-50931 Cologne, Germany

${ }^{2}$ CECAD - Cluster of Excellence

University of Cologne

D-50931 Cologne, Germany

${ }^{3}$ Center for Molecular Medicine Cologne (CMMC)

University of Cologne

D-50931 Cologne, Germany

\#these authors contributed equally

*Correspondence: mdenzel@age.mpg.de 


\section{Abstract}

2 Glucosamine feeding and genetic activation of the hexosamine biosynthetic pathway 3 (HBP) have been linked to improved protein quality control and lifespan extension in

4 various species. Thus, there is considerable interest in the potential health benefits of

5 dietary supplementation with glucosamine or other HBP metabolites in people. The

6 HBP is a sensor for energy availability and its activation has been implicated in tumor

7 progression and diabetes in higher organisms. As the activation of the HBP has been

8 linked to longevity in lower animals, it is imperative to explore the long-term effects of

9 chronic HBP activation in mammals, which has not been examined so far. To address

10 this issue, we activated the HBP in mice both genetically and through metabolite 11 supplementation, and evaluated metabolism, memory, and survival. GlcNAc supplementation in the drinking water had no adverse effect on weight gain in males but increased weight in young female mice. Glucose or insulin tolerance were not affected up to 20 months of age. Of note, we observed improved memory in the Morris water maze in young male mice supplemented with GlcNAc. Survival was not changed by GlcNAc supplementation. To assess the effects of genetic HBP activation we overexpressed the key enzyme GFAT1 as well as a constitutively activated point mutant form in all mouse tissues. We detected elevated UDP-GIcNAc levels in mouse brains, but did not find any effects on behavior, memory, or survival. Together, while dietary GIcNAc supplementation did not extend survival in mice, it positively affected memory and is generally well tolerated.

\section{Keywords}

23 Hexosamine biosynthetic pathway; GFAT1; mouse survival; metabolism; memory 


\section{Introduction}

25 The hexosamine biosynthetic pathway (HBP) is an anabolic pathway that consumes fructose-6-phosphate (Fru6P), glutamine, acetyl-CoA, and UTP to produce the high energy molecule uridine 5'-diphospho-N-acetyl-D-glucosamine (UDP-GlcNAc) (Figure 1a). The HBP thus requires carbohydrate, aminoacidic, lipidic, and nucleotide donors and is centrally positioned at a cross-roads of energy metabolism (Wells, Vosseller, and Hart 2003). The first step in the HBP is catalyzed by glutamine fructose6-phosphate amidotransferase (GFAT), that employs L-glutamine (GIn) as a nitrogen donor to convert Fru6P to D-glucosamine-6-phosphate (GlcN6P), diverting 2-3\% of cellular glucose into the HBP (Marshall, Bacote, and Traxinger 1991). Next, glucosamine 6-phosphate $\mathrm{N}$-acetyltransferase (GNA1) catalyzes the acetylation of GlcN6P using acetyl-CoA to produce N-acetyl-D-glucosamine-6-phosphate (GlcNAc6P); the phosphoglucomutase PGM3 then isomerizes GIcNAc6P to yield GIcNAc1P and finally, the phosphorylase UAP1 uses UTP to form UDP-GIcNAc. UDP-GIcNAc and its epimer UDP-GalNAc are precursors for biopolymer synthesis and for distinct glycosylation reactions including $\mathrm{N}$ - and O-linked glycosylation, and O-GIcNAcylation (Hanisch 2001; Hart 1997; Parodi 2000).

GFAT is the HBP's key enzyme and it is composed of two domains: the glutaminase domain that hydrolyses L-GIn to produce ammonia and L-glutamate (L-Glu), and the isomerase/transferase domain, which isomerizes Fru6P into Glc6P and catalyzes the transfer of ammonia to produce GlcN6P (Denisot, Goffic, and Badet 1991). In eukaryotes, GFAT1 is feedback inhibited by the end product of the HBP, UDP-GIcNAc. Our previous observations have delineated a number of single amino acid substitutions in GFAT1 that lead to increased activity through loss of UDP-GIcNAc feedback inhibition and altered domain interactions, resulting in elevated HBP flux and 
cellular UDP-GIcNAc accumulation (Denzel et al. 2014; Horn et al. 2020; Ruegenberg

et al. 2020, 2021). Two alternative reactions feed into the HBP and increase the flux, bypassing the finely regulated GFAT-mediated step: glucosamine (GIcN) can be incorporated through its phosphorylation via hexokinase to generate GlcN6P (Stocchi et al. 1981), whereas N-acetyl-D-glucosamine (GlcNAc) can be incorporated through 54 its phosphorylation via N-acetyl-D-glucosamine kinase producing GlcNAc6P (Weihofen et al. 2006). Overall, HBP activation can be achieved through the overexpression of the rate-limiting step enzyme GFAT1 or through the supplementation of the metabolites GlcN or GlcNAc.

O-GIcNAcylation is one mechanism linking UDP-GIcNAc availability, hence HBP activity, to protein function through post-translational modifications. Hyper-OGlcNAcylation is observed in many cancer types, suggesting that this modification is a key molecular event in tumor formation, progression, aggressiveness, and potentially a new cancer hallmark (Fardini et al. 2013). Elevated GFAT1 expression has been associated with poor overall survival in patients suffering hepatocellular carcinoma (Li et al. 2017) and, strikingly, GFAT inhibitors like the diazo-derivative of serine, azaserine, and 6-diazo-5-oxo-L-norleucine (DON) decrease HBP flux and exhibit anti-tumor activity (Lemberg et al. 2018). The HBP has also been shown to be involved in diabetes; HBP activation in young rats by GlcN infusion leads to insulin resistance and enhanced transcription factor glycosylation, resembling an aging phenotype (Einstein et al. 2008; Marshall, Bacote, and Traxinger 1991). Moreover,

70 GlcN impairs the GLUT4 glucose transporter (whose expression is stimulated by insulin), resembling an insulin-resistant state (Baron et al. 1995). These data highlight

72 the importance of monitoring potential adverse side effects upon chronic HBP activation. 
Although high HBP flux has been linked to cancer and diabetes, previous observations also support a plausible role in longevity and prevention of age-related pathologies (Denzel and Antebi 2015). The HBP is involved in protein quality control and its activation leads to improved protein homeostasis and lifespan extension in Caenorhabditis elegans: worms with GFAT-1 gain-of-function (GOF) mutations have increased UDP-GIcNAc/UDP-GalNAc levels and are long-lived (Denzel et al. 2014).

Strikingly, this lifespan extension is recapitulated by GlcNAc feeding in a dosedependent manner (Denzel et al. 2014). Elevating HBP metabolite levels through GFAT-1 GOF mutations or through GIcNAc supplementation enhances ER protein quality control, ER-associated protein degradation (ERAD), and autophagy (Denzel et al. 2014; Shintani, Kosuge, and Ashida 2018). Of note, worms overexpressing WT GFAT-1 or supplemented with GIcNAc activate the integrated stress response (ISR) (Horn et al. 2020). In mammalian Neuro-2a (N2a) cells, mild HBP activation through GFAT1 GOF mutation or through GIcNAc feeding likewise induces the ISR: the PERK branch is activated leading to increased phosphorylation of the a subunit of eukaryotic initiation factor 2 (elF2 $\alpha$ ) and upregulation of the stress master transcription factor ATF4 (Horn et al. 2020). In N2a cells, HBP activation has a protective role against proteotoxicity in a PERK- and autophagy-dependent manner and muscle cell-specific HBP activation rescues polyQ-mediated toxicity in worms, a process that depends on ATF4 (Horn et al. 2020). GlcN supplementation likewise induces ER stress in rat cells (Lombardi et al. 2012), and it leads to elF2 $\alpha$ phosphorylation through the PERK branch with a concomitant mRNA translation arrest (Kline et al. 2006). Overall, these data suggest a protective role of HBP activation in various species.

97 GlcN feeding extends lifespan in worms and mice, in a manner that is independent 98 from the HBP: GlcN supplementation impairs glucose metabolism and activates 
99 AMPK to promote mitochondrial biogenesis, which mimics low-carbohydrate diet

100 paradigms (Weimer et al. 2014). GlcN supplementation in mice leads to glycolysis

101 impairment as a rise in GlcN6P was observed (Weimer et al. 2014), a metabolite that

102 inhibits hexokinase (Silverman 1963). Strikingly, GlcN supplementation does not

103 increase the levels of UDP-GIcNAc in the mouse liver. Furthermore, silencing PGM3

104 within the HBP by RNAi does not impair GlcN-mediated lifespan extension in worms

105 (Weimer et al. 2014). Instead, GlcN treatment induces a metabolic switch towards

106 increased amino acid catabolism in mice (Weimer et al. 2014), supporting the HBP-

107 independent effect. Given the various observed effects of GlcN metabolism and HBP

108 activation, their role in mammalian life- and healthspan needs to be explored.

109 To investigate a possible modulatory role of the HBP in mammalian aging, we

110 employed distinct strategies to increase HBP activity and examined general behavior,

111 memory, and survival in mice. We found that GlcNAc supplementation in the drinking

112 water or overexpression of wildtype or GOF GFAT1 did not have adverse effects in

113 mice. Importantly, the interventions did not interfere with glucose metabolism. Mice

114 supplemented with dietary GlcNAc or overexpressing GFAT1 did not exhibit lifespan

115 extension. Nevertheless, we observed memory improvement in young male mice fed

116 with GlcNAc, suggesting a beneficial effect of HBP activation in mammals. 


\section{Results}

118 HBP pathway activation by GIcNAc feeding does not have adverse effects in

mice. To chronically activate the HBP, we supplemented the drinking water of

120 C57BL/6J mice with 1\% GIcNAc starting at 8 weeks of age. We first tested whether

121 GlcNAc feeding might lead to adverse reactions. Regarding water intake, females consumed more GlcNAc-supplemented water compared to controls at 9 months of age; males, however, did not show any difference in water consumption (Figure 1b).

The drinking volume of around $4 \mathrm{ml}$ per day corresponds to a GlcNAc consumption of

1.5-2 $\mathrm{ng}^{*} \mathrm{~g}^{-1}$ body weight per day (Figure 1c). Importantly, GlcNAc supplementation in the drinking water had no effect on food intake (Figure S1a). We used LC-MS to quantify UDP-HexNAc levels (combined UDP-GIcNAc and UDP-GalNAc) in brain lysates from 9 months old mice to test effects of dietary supplementation. Surprisingly, after 7 months of GIcNAc feeding, the UDP-HexNAc levels were not affected in mouse brains (Figure 1d); similarly, liver samples did not show increased levels of UDP-HexNAc (data not shown). Because elevated flux through the HBP has been associated with insulin resistance and diabetic complications (Han, Chen, and Holloszy 2003; Rossetti et al. 1995), we tested the effect of chronic GlcNAc supplementation on glucose utilization by measuring blood glucose clearance and insulin-stimulated glucose utilization. Chronic GlcNAc intake had no detectable effect on either blood glucose clearance or insulin response in 10 months old mice

137 (Figure 1e,f and Figure S1c), or 20 months old mice (Figure S1b,c). In 4 months old females, however, we observed slightly higher blood glucose levels 30 and 60 min after the injection of insulin (Figure S1c). Nevertheless, overall, we conclude that any difference in body weight, GlcNAc-supplemented female mice showed increased 
body weight compared to controls (Figure 1g). Indirect calorimetric measurements revealed an increased respiratory exchange ratio (RER) in 9 months old female mice during the day, while no difference was detected in age-matched male mice (Figure S1d). This increased RER, as well as the effects on body weight, might be caused by the elevated GlcNAc consumption (Figure 1b,c), which was also sex-specific. Despite these effects, female mice did not display changes in insulin signaling after 4 months.

148 Therefore, overall, our data suggest that chronic GlcNAc supplementation does not have negative side effects in mice.

GIcNAC supplementation does not affect coordination or neuromuscular

function. Having excluded negative side effects of dietary GICNAc supplementation on the metabolic health of mice, we next aimed to test possible effects of GlcNAc supplementation on coordination and basic neuromuscular function. To this end, the fitness of mice at 6 months of age was analyzed using the rotarod, and by assessing grip strength and treadmill endurance. Of note, GlcNAc supplementation did not affect grip strength (Figure 2a-b), rotarod performance (Figure 2c), or forced maximal endurance on a treadmill (Figure 2d). Thus, fitness and locomotion of mice were not changed by GlcNAc supplementation.

As GIcNAc supplementation extends C. elegans lifespan (Denzel et al. 2014) and showed no adverse effects in mice, we supplemented mice of both sexes with GIcNAc in the drinking water from week 8 until they died to assess the effect on mammalian

162 lifespan. GlcNAc feeding did not extend mouse survival (Figure 2e). Importantly however, survival was not reduced by GlcNAc supplementation. In sum, these data support that chronic HBP activation by GICNAc feeding does not have adverse side effects on general fitness and health in mice. 

activity or exploratory behavior as measured by the distance, speed, and percentage

172 of distance spent in the center of the open field at 6 months of age (Figure 3a-b;

173 Figure S2a). Additionally, analysis of the home cage activity in metabolic cages did not reveal differences caused by GlcNAc supplementation (Figure S2b). Thus, locomotion and exploratory behavior were not affected by GlcNAc treatment. In the Morris water maze, the animals were trained for consecutive 5 days, and after

177 the last session, memory was assessed by removal of the hidden platform. During 178 training, both distance and searching time (latency) decreased progressively 179 (Figure S2c-d), while swimming speed remained constant (Figure S2e). These data 180 suggest that the mice learned to find the hidden platform. There was no significant 181 difference between GlcNAc-fed animals compared to controls (Figure S2c-e) during 182 the training period. Still, GIcNAc-supplemented male mice tended to swim shorter distances and to spend less time in the water from day 2 on (Figure S2c-d). During the test, there was no difference in the time mice spent in the target quadrant upon removal of the platform (Figure 3c), strikingly, however, the latency of the first platform visit (defined as the time needed until the mice cross the area where the platform used 187 to be) was strongly decreased in GlcNAc-fed males (Figure 3d). Furthermore, the number of platform visits (defined as the number of times the mice cross the area where the platform used to be) was increased in GlcNAc-fed male mice (Figure 3e).

190 Overall, our observations suggest that GlcNAc feeding improves memory of young 191 male mice. 
HBP activation by overexpression of WT or G451E huGFAT1 does not influence

body weight in mice. To corroborate the data obtained upon dietary GlcNAc

supplementation, we pursued a parallel approach to genetically activate the HBP by

overexpressing N-terminally FLAG-HA tagged human GFAT1 (huGFAT1) in all mouse

tissues. The Rosa26 locus was engineered to contain an expression construct

composed of a loxP-flanked transcription termination cassette upstream of the

huGFAT1 open reading frame (huGFAT1 $\mathrm{wt} \mathrm{tg}^{+/-}$, Figure $4 \mathrm{a}$ ). These mice were

crossed with transgenic CMV-cre ${ }^{+/-}$females, to obtain huGFAT1 overexpressing

animals (huGFAT1 wt OE, Figure 4a). The functionality of the cassette was confirmed

by Western blot analysis (Figure 4b): In fibroblasts isolated from huGFAT1 wt $\operatorname{tg}^{+/-}$

newborn mice HA expression was not detectable due to the lack of cre recombinase expression. Endogenous GFAT1 expression was comparable to WT and CMW-cre ${ }^{+/-}$ fibroblasts. huGFAT1 wt OE fibroblasts in which the GFAT1 transgene and cre recombinase were co-expressed, displayed elevated GFAT1 and HA expression, demonstrating successful expression of the transgene. Accordingly, UDP-HexNAc levels were comparable in WT, huGFAT1 $\mathrm{wt} \mathrm{tg}^{+/-}$and CMV-cre ${ }^{+/-}$fibroblasts and about 2-fold elevated by GIcNAc supplementation and huGFAT1 wt OE (Figure 4c). As

(Figure 4d) and UDP-GalNAc (Figure S3a) in the brains of 3 months old male and

211 female mice compared to brains of WT and CMV-cre ${ }^{+/-}$mice. Body weight analysis over 27 months indicated that there was no effect of huGFAT1 wt overexpression compared to CMV-cre ${ }^{+/-}$mice in both sexes (Figure 4e). However, cre expression

214 slightly reduced body weight in male mice compared to WT controls (Figure 4e). 
substitution is associated with C. elegans longevity (Denzel et al. 2014) and confers a drastically reduced sensitivity to UDP-GIcNAc feedback inhibition (Ruegenberg et al. 2020). huGFAT1 G451E overexpression resulted in elevated levels of both UDP-GICNAc and UDP-GalNAc in the brains of 3 months old male and female mice

221 (Figure S3b-c). Body weight was not affected by huGFAT1 gof OE between 12 and 27 months of age compared to CMV-cre ${ }^{+/-}$mice in both sexes (Figure S3d). Together, these data suggest successful genetic HBP activation without detrimental effects on overall health as assessed by body weight analysis in huGFAT1 wt and gof OE mice. As we observed no relevant differences between huGFAT1 wt or G451E gof overexpression, we focused the remainder of the analyses on the huGFAT1 wt OE mice.

HBP activation by huGFAT1 WT OE does not influence coordination,

neuromuscular function, memory, or lifespan. To study coordination and basic neuromuscular function upon genetic HBP activation, experiments using rotarod and treadmill were performed and grip strength was measured using all genotypes at three different time points $(3-4,15-16$, and $21-22$ months of age). In line with the results using dietary GlcNAc supplementation, genetic HBP activation did not affect grip strength (Figure 5a, Figure S4a), rotarod performance (Figure 5b), or forced maximal endurance on a treadmill (Figure 5c). To assess the effect of genetic HBP activation on survival, lifespan experiments with transgenic mice of both sexes were performed. Survival of the huGFAT1 wt or gof OE mice was indistinguishable from the corresponding genetic controls (Figure 5d, Figure S4b).

We assessed both general locomotor activity and exploratory behavior in the open was influenced by cre expression, since CMV-cre ${ }^{+/-}$and huGFAT1 wt OE mice moved 
more and faster compared to WT controls (Figure 6a, Figure S5a). This effect was

measured by the percentage of distance spend in the center of the open field was similar in all genotypes (Figure 6b). Memory and learning were tested in both $Y$ maze and Morris water maze. In the $Y$ maze, alternations, i.e. how often a mouse chooses to explore a new arm over the same arm, did not change among the different genotypes, suggesting no differences in exploratory behavior and spatial working memory (Figure 6c). The distance covered in the $Y$ maze was comparable across all genotypes in females, while it was increased in males upon cre expression (Figure $\mathrm{S} 5 \mathrm{~b})$. In the Morris water maze, there was no difference in learning in 4 months old mice, since all genotypes of both sexes showed a similar improvement regarding distance and latency during the training sessions (Figure S5c-d). The speed of the mice was slightly decreased on day 5 compared to day 1 , however, this effect was similar in all genotypes (Figure S5e). Memory, which was tested by the removal of the platform, was unchanged, since the time spent in the target quadrant (Figure 6d), the the latency of the first platform visit (Figure 6e), and the number of platform visits (Figure 6f) were similar in all genotypes. Altogether, and in contrast to dietary GIcNAc supplementation, genetic HBP activation did not improve memory in mice. Overall, results obtained using the genetic model support the conclusion that HBP activation does not have adverse effects in mice. While lifespan was not affected in any of the male mice. 


\section{Discussion}

In this study, we delineate the effect of HBP activation on general behavior, memory, of mice. While fitness, locomotion, behavior, and survival were not changed by HBP activation, we observed memory improvement in young male mice fed with GlcNAc, suggesting a beneficial effect of HBP activation in mammals.

GlcNAc is rapidly absorbed and enters the systemic circulation when fed via the drinking water: experiments using ${ }^{13} \mathrm{C}_{6}$-GlcNAc indicate that GlcNAc peaks in the serum after 30 min of gavage and is cleared from the circulation after $2-3 \mathrm{~h}$; by this time, UDP- ${ }^{13} \mathrm{C}_{6}-$ GlcNAc can be detected in liver, kidney, and spleen (Ryczko et al. 2016). ${ }^{13} \mathrm{C}_{6}-\mathrm{GlcNAc}$, orally supplemented at a concentration of $0.1 \%$, has been shown analysis identified UDP-[U $\left.{ }^{13} \mathrm{C}\right]-\mathrm{HexNAc}$ in the brain of adult females (Sy et al. 2020). These data indicate that GlcNAc fed via the drinking water contributes to the UDP-GIcNAc pool in brain and liver. While we could show in this (Figure 4c) and in previous studies that GIcNAc supplementation is sufficient to increase UDP-GIcNAc levels in worms and in cells (Denzel et al. 2014; Horn et al. 2020) the levels of UDP-HexNAc were not increased in the brain or liver of mice fed with $1 \%$ GlcNAc after 7 months of feeding (Figure 1d). Previously, it has been shown that GlcNAc feeding elevates hepatic UDP-HexNAc levels by around 25\% (Ryczko 2016). Given this minor effect and the fast clearance of GlcNAc from the circulation, the timing of sample collection might have influenced our results: The mice were sacrificed during the day, when they drink less. Additionally, we analyzed steady-state UDP-HexNAc levels; thus, we cannot exclude elevated HBP flux upon dietary GIcNAc supplementation. 
Dietary GIcNAc supplementation has previously been shown to increase body weight: in hepatic UDP-GIcNAc levels (Ryczko et al. 2016). In contrast, 25 months old mice fed for about 8 months with $10 \mathrm{~g} \mathrm{GlcN} \mathrm{per} \mathrm{kg}$ of diet did not show an increase in body weight (Weimer et al. 2014). Of note, UDP-GlcNAc levels were not elevated in these animals, suggesting that body weight and UDP-GIcNAc levels correlate. In this study, age (Figure 1g). In contrast, females fed with 1\% GIcNAc showed significantly increased body weight up to 10 months of age; later in life, the body weight was slightly, but not significantly elevated (Figure 1g). Interestingly, 9 months old females consumed more GlcNAc-containing water (Figure 1b); however, they did not display significantly elevated UDP-HexNAc levels (Figure 1d). Thus, GlcNAc intake rather than UDP-HexNAc levels correlated with body weight in this study.

HBP activation has also been linked to impaired insulin signaling: adipocytes treated with GlcN become insulin resistant (Marshall, Bacote, and Traxinger 1991) and rats infused with GlcN also exhibit insulin resistance (Virkamaki 1997), similar to GlcNtreated mice (Weimer et al. 2014). In our experiments, GlcNAc feeding did not alter the response to insulin up to 20 months of age (Figure S1c). In line with our results, although in a different animal model using pellet-based supplementation, rats exposed to $5 \%$ of GlcNAc for 1 year did not exhibit changes in the basal levels of serum glucose

311 (Takahashi et al. 2009). Overall, these data suggest that GlcN-mediated HBP 312 activation impairs insulin signaling, whereas dietary GlcNAc supplementation does not.

314 In worms, HBP activation by GIcNAc supplementation as well as GOF mutations in 
315 GFAT-1 extend lifespan (Denzel et al. 2014). In this study, the lifespan of mice fed

316 with 1\% GIcNAc was indistinguishable from controls (Figure 2e). However, based on

317 the unaltered UDP-HexNAc levels in the brains of GIcNAc-fed animals, the HBP

318 activity achieved here might not be sufficient to extend lifespan. Alternatively, the

319 mechanism underlying the lifespan extension might not be conserved between worms

320 and mammals. Importantly, GlcNAc feeding did not shorten mammalian lifespan and

321 the overall health of the mice was not negatively affected by the treatment.

322 Putative LOF mutations in GFAT1 are associated with congenital myasthenic 323 syndrome, which is characterized by defective neuromuscular junctions (Senderek et

324 al. 2011). Accordingly, activating the HBP pathway by GlcNAc feeding might have beneficial effects on neuromuscular function. However, we did not observe differences in coordination and fitness upon GlcNAc feeding (Figure 2a-d). While spontaneous locomotor activity and exploratory behavior were unchanged in GlcNAc-fed mice compared to controls (Figure 3a-b), GlcNAc-fed young males exhibited memory improvement in the Morris water maze (Figure 3d-e). Of note, various studies have demonstrated the contribution of myelin formation to memory consolidation and recall

331 (Pan et al. 2020; Steadman et al. 2020). Strikingly, the HBP has a primary role in myelination: genetic or toxin-driven blockage of $\mathrm{N}$-glycan branching induces demyelination, a phenotype that can be rescued by oral GlcNAc feeding (Sy et al. 2020). Furthermore, the inducible deletion of O-GlcNAc transferase -the enzyme responsible for adding GlcNAc to substrate proteins- results in learning and memory deficits (Wheatley et al. 2019). Thus, usage of the HBP's end-product for posttranslational modifications plays an important role in memory. Overall, these data suggest that the observed memory improvement in young males fed with $1 \%$ GlcNAc could be a consequence of increased myelination driven by elevated HBP activity. 
340 Although the steady-state UDP-HexNAc levels in GlcNAc-fed mice were

341 indistinguishable from controls (Figure 1d), we cannot exclude enhanced HBP flux.

342 To corroborate our findings obtained with dietary GlcNAc supplementation, we

343 additionally analyzed a model of genetic HBP activation. While ubiquitous

344 overexpression of wt GFAT1 led to a marked increase in GFAT1 protein (Figure 4b), brain UDP-HexNAc levels were only slightly elevated in 3 months old mice (Figure 4d,

346 Figure S3a). Moreover, the overexpression of gain-of-function GFAT1 G451E, which

347 displays reduced sensitivity to UDP-GlcNAc feedback inhibition (Ruegenberg 2020),

348 activated the HBP to a similar extent as wt GFAT1 OE (Figure S3b-c). Potentially, the

349 N-terminal tag interfered with GFAT1 activity, as previously described (Olchowy et al.

350 2006). Nevertheless, the mice had around 2-fold increased UDP-HexNAc levels, demonstrating successful HBP activation.

GFAT1 overexpression in different tissues (namely skeletal muscle, fat, liver, and $\beta$ cells) leads to disease-like metabolic states such as insulin resistance, obesity, hyperlipidemia, and impaired glucose metabolism (Hebert et al. 1996; McClain et al. 2002; Veerababu et al. 2000). While GFAT1 overexpression in liver has been associated with overweight (Veerababu et al. 2000), our results indicate that both GFAT1-overexpressing mice and controls have similar body weight profiles over the lifetime of the animals (Figure $4 e$ and S3d). The impact of GFAT1 overexpression on coordination and neuromuscular function has never been approached before; strikingly, our results indicate that GFAT1 overexpression did not affect fitness, spontaneous activity, or exploratory behavior (Figure 5a-c, Figure S4a and Figure 6ab). In contrast to GICNAc feeding, GFAT1 overexpression did not enhance learning and memory formation compared to controls (Figure 6c-f and Figure S5c-e). These data suggest that the GlcNAc-mediated effect on memory might be independent of 
HBP activity. Of note, GIcN supplementation has been previously shown to impair glucose metabolism and to promote mitochondrial biogenesis via activation of AMPK

367 (Weimer et al. 2014). Therefore, investigating the role of these mechanism in the

368 GlcNAc-mediated memory improvement would be of interest in the future.

369 Finally, neither the overexpression of wt GFAT1 nor the G451E mutant had an impact

370 on mouse survival (Figure 5d and Figure S4b). In contrast to GlcNAc-fed mice

371 (Figure 1d), the transgenic mice did show increased UDP-GIcNAc levels (Figure 4d

372 and Figure S3a-c). Thus, while HBP activation results in longevity in C. elegans

373 (Denzel et al. 2014), it was not sufficient to extend lifespan in mice. In this study, either

374 HBP activation did not reach sufficient UDP-GIcNAc levels for lifespan extension or

375 the lifespan-modulating effects of HBP activation in the worm are caused by nonconserved mechanisms.

377 In sum, this study dissects the effects of dietary GlcNAc supplementation and genetic

378 HBP activation on murine health, behavior, memory, and lifespan. Despite being 379 linked to diabetes, obesity, and cancer, these interventions did not have adverse 380 effects in mice. Instead, we show that GlcNAc feeding increases memory formation of 381 young male mice. Of note, this effect might be independent of HBP activity, since we 382 did not observe changes in memory upon genetic HBP activation. In the future, HBP383 independent effects of GlcNAc supplementation should be tested in the context of 384 learning and memory. 
Experimental procedures

386

387

388

389

390

391

392

393

394

395

396

397

398

399

400

401

402

403

404

405

406

407

\section{Mouse husbandry}

Animals were housed on a 12:12 h light:dark cycle with ad libitum access to food under pathogen-free conditions in individually ventilated cages. All animals were kept in C57BL/6J background. Animal care and experimental procedures were in accordance with the institutional and governmental guidelines.

\section{GIcNAc feeding}

Mice were fed with control water or water containing $1 \%$ GlcNAc (w/v) beginning at 8 weeks of age. GlcNAc was provided by Wellesley Therapeutics Inc., Toronto, Canada.

\section{Generation of transgenic mice}

Generation of transgenic GFAT1 mice was performed by Taconic Biosciences (Cologne, Germany). An expression cassette was inserted in the Rosa26 locus using recombination-mediated cassette exchange in embryonic stem cells. The cassette encodes a loxP-flanked transcription termination cassette upstream of the human GFAT1 (huGFAT1) open reading frame (conditional knock-in allele, huGFAT1 wt/gof $\left.\operatorname{tg}^{+/-}\right)$. Upon cre-mediated deletion of the transcription termination cassette, huGAFT1 is expressed under the control of the chicken $\beta$-actin promoter, resulting in its overexpression (constitutive knock-in allele, huGFAT1 wt/gof OE). huGFAT1 is N-terminally tagged with FLAG-HA (HA: hemagglutinin; for further information, see Figure 4). Mice expressing the cre recombinase under the control of the human cytomegalovirus minimal promoter $\left(\mathrm{CMV}_{-} \mathrm{cre}^{+/}\right)$were purchased from Charles River Laboratories (Sulzfeld, Germany). 


\section{Breeding of transgenic mice}

409 For breeding, huGFAT1 wt/gof $\mathrm{tg}^{+/-}$males were crossed with transgenic CMV-cre ${ }^{+/-}$

410 females. Both transgenes were maintained in a heterozygous state. Since the CMV

411 promoter is active before implantation during early embryogenesis (Schwenk, Baron,

412 and Rajewsky 1995), animals expressing the cre recombinase and carrying the

413 GFAT1 transgene were considered to overexpress huGFAT1 in all tissues. The

414 offspring was genotyped as described below.

Tissue collection

416 Mice were sacrificed by cervical dislocation. Brains from 3 months old (genetic model)

417 or 9 months old (GlcNAc feeding) male and female mice were dissected. Cerebral

418 hemispheres were snap frozen in liquid nitrogen and stored at $-80^{\circ} \mathrm{C}$ until further use.

\section{Lifespan analysis}

420 Lifespan analysis was performed with 69 female and 82 male mice per

421 genotype/condition. The general health of the mice was monitored regularly and the

422 mice were euthanized when necessary, according to a pre-defined score sheet. The

423 lifespan analysis comprises all mice that died a natural death or were euthanized. The

424 body weight of the mice was analyzed every other week (GlcNAc feeding) or every 3

425 months (huGFAT1 wt/gof OE). To exclude an effect on lifespan, no other experiments

426 were performed with these mice.

\section{Metabolic cages}

428 Indirect metabolic analyses were performed in singly housed 3 months and 9 months 429 old mice for $48 \mathrm{~h}$ using metabolic cages (Phenomaster, TSE Systems). Mice were 430 habituated to the cages for $24 \mathrm{~h}$ before the measurements. Analysis of the air before 431 and after passing the cages allowed calculation of oxygen consumption and carbon 
432

433

434

435

436

437

438

439

440

441

442

443

444

445

446

447

448

449

450

451

452

dioxide production. These values were used to determine the metabolic respiratory quotient of these mice. Additionally, the spontaneous locomotor activity, as well as food and water consumption of the mice were monitored.

\section{Glucose and insulin tolerance tests}

Glucose and insulin tolerance was determined in 4 months, 10 months, and 20 months old mice upon GlcNAc feeding. For the glucose tolerance test, mice were fasted for $16 \mathrm{~h}$ with full access to drinking water. $2 \mathrm{~g}$ glucose per $\mathrm{kg}$ body weight were injected intraperitoneally. To monitor blood glucose concentration, a blood sample was taken before and 15, 30, 60, and 120 min after the glucose injection. For the insulin tolerance test, $0.75 \mathrm{U}$ insulin per $\mathrm{kg}$ body weight were injected intraperitoneally. To monitor blood glucose concentration, a blood sample was taken before and 15, 30, and 60 min after the insulin injection. Blood glucose concentration was measured using an automatic glucose monitor (Accu-Check Aviva, Roche).

\section{Grip strength measurements}

Grip strength measurements were performed at 6 months of age (GlcNAc feeding) or with 3,15 , and 22 months of age (huGFAT1 wt OE). The mouse is holding a trapeze while being pulled backward until the pulling force is bigger than the grip strength of the mouse (2 paws). The maximal grip strength of the mouse is recorded automatically. Alternatively, the mouse is placed on a grid and the grasping applied by the mouse while being pulled backwards is measured (4 paws). The tests were repeated 5 times per mouse per day and the mean of the 5 trials is plotted.

\section{Open field}

Open field analyses were performed with 6 months of age (GIcNAc feeding) or with 3, 15 , and 21 months of age (huGFAT1 wt OE). The mice were placed in a $50 \times 50 \times 40 \mathrm{~cm}$ 
big box for $10 \mathrm{~min}$. The spontaneous locomotor and explorative activity, as well as the

457 speed were analyzed by tracking the movement of the mice.

\section{$458 \quad$ Rotarod}

459 Rotarod analyses were performed at 6 months of age (GlcNAc feeding) or with 3, 15, 460 and 21 months of age (huGFAT1 wt OE). The rotation speed of the rod constantly 461 increased from 5 to $40 \mathrm{U} / \mathrm{min}$ within $5 \mathrm{~min}$. The time until the mice fell of the rod was measured. The experiment was performed twice per day on two or four consecutive days for GIcNAc feeding and huGFAT1 wt OE mice, respectively. The average time on the rod of the two runs on the last day of the experiment is plotted.

\section{Treadmill}

Treadmill analyses were performed at 6 months of age (GIcNAc feeding) or with 4, 16, and 22 months of age (huGFAT1 wt OE). After an adaption phase of $5 \mathrm{~min}$, the treadmill was started with a speed of $0.1 \mathrm{~m} / \mathrm{s}$. This speed was maintained for $10 \mathrm{~min}$ before it constantly increased to $1.3 \mathrm{~m} / \mathrm{s}$ within $60 \mathrm{~min}$. An electric shock of $0.3 \mathrm{~mA}$ was given whenever the mouse stayed at the end of the treadmill for more than $2 \mathrm{sec}$.

471 The experiment was stopped when a mouse received three consecutive shocks. The 472 cumulative distance was analyzed for each mouse.

\section{$473 \quad$ Y maze}

474 Y maze analyses were performed with 3, 15, and 21 months of age (huGFAT1 wt OE).

475 The mouse is placed in one arm of the $Y$ maze and can explore the environment for

$4765 \mathrm{~min}$. The activity of the mouse is tracked. Distance and alternations are measured

477 for each mouse. The alterations describe how often a mouse chooses to explore a 478 new region over the same region. 


\section{$479 \quad$ Water maze}

480 Water maze analyses were performed with 4 months of age (GlcNAc feeding and huGFAT1 wt OE). The mice were placed in a basin filled with stained water, in which a platform was hidden $1-2 \mathrm{~cm}$ below the surface of the water. Since the mice want to avoid swimming, they will learn to find the hidden platform during training sessions. Here, the mice were trained for five consecutive days on which they performed four trials per day, with a maximum duration of $60 \mathrm{~s}$ per trial. The average of the four trials this final test, the memory of the mice was analyzed. The mice were placed in the basin for $60 \mathrm{~s}$ and their preference for the quadrant which previously contained the platform, as well as the latency of the first visit and the number of visits of the former platform was analyzed.

\section{Isolation of mouse genomic DNA from ear clips}

492 Ear clips were taken by the Comparative Biology Facility at the Max Planck Institute for Biology of Ageing (Cologne, Germany) at weaning age (3-4 weeks of age) and stored at $-20^{\circ} \mathrm{C}$ until use. $150 \mu \mathrm{ddH} 2 \mathrm{O}$ and $150 \mu$ directPCR Tail Lysis reagent

495 (Peqlab) were mixed with $3 \mu$ proteinase $\mathrm{K}(20 \mathrm{mg} / \mathrm{ml}$ in $25 \mathrm{mM}$ Tris- $\mathrm{HCl}, 5 \mathrm{mM}$ $\mathrm{Ca} 2 \mathrm{Cl}, \mathrm{pH}$ 8.0, Sigma-Aldrich). This mixture was applied to the ear clips, which were then incubated at $56^{\circ} \mathrm{C}$ overnight (maximum $16 \mathrm{~h}$ ) shaking at $300 \mathrm{rpm}$. Proteinase $\mathrm{K}$ was inactivated at $85^{\circ} \mathrm{C}$ for $45 \mathrm{~min}$ without shaking. The lysis reaction $(2 \mu \mathrm{l})$ was used for PCR without further processing. For genotyping of newborn pups, the tail tip was lysed in $300 \mu$ directPCR Tail Lysis reagent containing $3 \mu$ l proteinase $\mathrm{K}$. 


\section{Genotyping PCR}

502 For genotyping of mouse genomic DNA DreamTaq DNA polymerase (ThermoFisher

503 Scientific) was used. The presence of the cre transgene was determined using 504 Cre_fwd (GCCAGCTAAACATGCTTCATC) and Cre_rev

505 (ATTGCCCCTGTTTCACTATCC). The primer targeting huGFAT1 (huGFAT1_fwd 506 CGGTGGAGGTTACCCATACG; huGFAT1_rev CGAGCTTGGCAATTGTCTCTG)

507 detected presence of the transgene without distinction of zygosity. The product amplified using oIMR7338 and olMR7339 served as internal control for huGFAT1 and

509 cre transgene detection. Using the huGFAT1_R26 PCR (3224_35

510 TTGGGTCCACTCAGTAGATGC; 1114_1 CTCTTCCCTCGTGATCTGCAACTCC;

511 1114_2 CATGTCTTTAATCTACCTCGATGG ), the wildtype Rosa26 locus and

512 the targeted Rosa26 locus could be distinguished.

\section{Isolation and maintenance of primary fibroblasts}

514 For fibroblast isolation newborn mice (P0-P3, both sexes) were sacrificed by

515 decapitation. The corpus was incubated in 50\% betaisodona/PBS (Mundipharma

$516 \mathrm{GmbH}$ ) for $30 \mathrm{~min}$ at $4^{\circ} \mathrm{C}$ before being washed in different solutions for 2 min each:

517 PBS (ThermoFisher Scientific), 0.1\% octenidin in ddH2O (Serva Electrophoresis),

518 PBS, 70\% ethanol, PBS, antibiotic-antifungal-solution in PBS (ThermoFisher

519 Scientific). Tail and legs were removed and the tail tip was used for genotyping.

520 Complete skin was separated from the body and incubated in $2 \mathrm{ml}$ dispase II solution

521 (5 mg/ml in $50 \mathrm{mM}$ HEPES/KOH pH 7.4, $150 \mathrm{mM} \mathrm{NaCl}$; Sigma-Aldrich) over night at

$5224^{\circ} \mathrm{C}$. The epidermis was separated from the dermis as a sheet. The dermis was

523 minced into small pieces using scalpels and transferred to a falcon tube containing

524 collagenase $(400 \mathrm{U} / \mathrm{ml}$ in $50 \mathrm{mM}$ Tris base, $5 \mathrm{mM} \mathrm{CaCl}$, $\mathrm{pH}$ 7.4; Sigma-Aldrich). The 525 samples were incubated at $37^{\circ} \mathrm{C}$ for $1.5 \mathrm{~h}$ and mixed regularly. Next, the suspension 
was filtered through a $70 \mu \mathrm{m}$ cell strainer, which was washed with DMEM afterwards.

527 The cells were centrifuged for $10 \mathrm{~min}$ at $1000 \mathrm{rpm}$. The pellet was resuspended in

528 DMEM (4.5 g/L glucose, $10 \%$ fetal bovine serum and penicillin/streptavidin; all

529 ThermoFisher Scientific) and the cells were seeded on non-coated tissue culture

530 plates. The cells were grown at $37^{\circ} \mathrm{C}$ in $5 \% \mathrm{CO}_{2}$.

\section{Western Blot analysis}

532 Protein concentration of cell lysates was determined using the Pierce ${ }^{\mathrm{TM}}$ BCA protein assay kit according to manufacturer's instructions (ThermoFisher Scientific). Samples were subsequently subjected to SDS-PAGE and blotted on a nitrocellulose membrane. The following antibodies were used in 5\% low-fat milk (Carl Roth) or 1\% bovine serum albumin (BSA; Carl Roth) in TBS-Tween buffer (25 mM Tris base,

$537150 \mathrm{mM} \mathrm{NaCl}, 2 \mathrm{mM} \mathrm{KCl}, \mathrm{pH} 7.4 ; 0.05 \%$ Tween-20 (w/v)) over night at $4^{\circ} \mathrm{C}$ : GFPT1

538 (rabbit, Abcam, EPR4854, 1:1.000 in BSA), hemagglutinin (HA; rat, Roche

539 Diagnostics $\mathrm{GmbH}, 3 \mathrm{~F} 10,1: 1.000$ in milk), $\beta$-actin (mouse, Cell Signaling

540 Technologies, 8H10D10, 1:25.000 in milk). After incubation with HRP-conjugated

541 secondary antibody (Invitrogen, 1:5000), the blot was developed using ECL solution

542 (Merck Millipore). Bands were detected on a ChemiDoc MP Imaging System (Bio-Rad 543 Laboratories).

\section{Metabolite analysis}

545 Determination of UDP-HexNAc levels

546 Brains were collected from 9 months old mice, cut in half, and snap frozen in liquid

547 nitrogen. For metabolite extraction, $250 \mu \mathrm{dddH} 2 \mathrm{O}$ were added to the hemibrains and

548 the tissue was disrupted using a dounce homogenizer. The samples were subjected 549 to four freeze/ thaw cycles (liquid nitrogen/ $37^{\circ} \mathrm{C}$ water bath). Next, the protein 
551 Scientific). $200 \mu \mathrm{l}$ with a protein concentration of $1 \mu \mathrm{g} / \mu \mathrm{l}$ were mixed with $1 \mathrm{ml}$

552 chloroform:methanol (1:2) and incubated on a nutator mixer for $1 \mathrm{~h}$ at RT. After

553 centrifugation for $5 \mathrm{~min}$ at full speed, the supernatant was transferred to a glass vial.

554 The liquid was evaporated in an EZ-2 Plus Genevac centrifuge evaporator (SP 555 Scientific) with the following settings: time to final stage $15 \mathrm{~min}$, final stage time $4 \mathrm{~h}$,

556 low boiling point mixture. After evaporation, the samples were stored at $-20^{\circ} \mathrm{C}$ until

557 further use.

558 Absolute UDP-HexNAc levels were determined using an Acquity UPLC connected to

559 a Xevo TQ Mass Spectrometer (both Waters) and normalized to total protein content.

560 The measurements and subsequent analysis were performed as previously described

561 (Denzel et al. 2014).

562 Determination of UDP-GIcNAc and UDP-GalNAc levels

563 Brains were collected from 3 months old mice, cut in half, and snap frozen in liquid

564 nitrogen. Tissue was disrupted using a TissueLyser II (Qiagen) at 20-25 Hz. The

565 powder was transferred to a fresh tube and subjected to metabolite extraction.

566 Metabolite extraction was performed using $80 \%$ methanol. After vortexing, the

567 samples were incubated at $-20^{\circ} \mathrm{C}$ for $30 \mathrm{~min}$. Afterwards, samples were incubated on

568 an orbital mixer at $5^{\circ} \mathrm{C}$ for $30 \mathrm{~min}$. The samples were centrifuged for $5 \mathrm{~min}$ at full speed

569 and $4^{\circ} \mathrm{C}$. The supernatant was transferred to a fresh tube and the pellet was used for

570 protein extraction with $0.5 \%$ SDS. The supernatant was evaporated in a SpeedVac 571 concentrator at $25^{\circ} \mathrm{C}$.

572 The metabolite analysis was conducted using a Dionex ICS-5000 anion exchange 573 chromatography (ThermoFisher Scientific). Separation was performed with a Dionex 574 Ionpac AS11-HC column (2 $\mathrm{mm} \times 250 \mathrm{~mm}, 4 \mu \mathrm{m}$ particle size, Thermo Fisher) at $30^{\circ} \mathrm{C}$. A 
575 guard column, Dionex Ionpac AG11-HC b $(2 \mathrm{~mm}$ x $50 \mathrm{~mm}, 4 \mu \mathrm{m}$ particle size,

576 ThermoFisher Scientific), was placed before the separation column. The eluent (KOH)

577 was generated by a $\mathrm{KOH}$ cartridge using $\mathrm{ddH}_{2} \mathrm{O}$. A gradient was used for the separation

578 at a flow rate of $0.380 \mathrm{ml} / \mathrm{min}: 0-8 \min 30 \mathrm{mM} \mathrm{KOH}, 8-12 \min 35-100 \mathrm{mM} \mathrm{KOH}, 12-15 \mathrm{~min}$

$579100 \mathrm{mM} \mathrm{KOH}, 15-19 \mathrm{~min} 30 \mathrm{mM} \mathrm{KOH}$. A Dionex suppressor AERS 500 (2 mm) was used

580 for the exchange of $\mathrm{KOH}$ and operated with $95 \mathrm{~mA}$ at $17^{\circ} \mathrm{C}$. The suppressor pump flow

581 was set to $0.6 \mathrm{ml} / \mathrm{min}$. Samples were diluted in $\mathrm{ddH}_{2} \mathrm{O}$ and injected from a tempered

582 autosampler $\left(8^{\circ} \mathrm{C}\right)$ using full loop mode $(10 \mu \mathrm{l})$. The Dionex ICS-5000 was connected to a

583 XevoTM TQ mass spectrometer (Waters) and operated in negative ESI MRM (multi

584 reaction monitoring) mode. The source temperature was set to $150^{\circ} \mathrm{C}$, the desolvation

585 temperature was set to $350^{\circ} \mathrm{C}$ and desolvation gas was set to $650 \mathrm{l} / \mathrm{h}$, while cone gas was

586 set to $50 \mathrm{l} / \mathrm{h}$. The MRM transition $606.10 \rightarrow 158.80$ was used for quantification of

587 UDP-GIcNAc and UDP-GalNAc. An external standard calibration curve was prepared from

58850 to $1000 \mathrm{ng} / \mathrm{ml}$ UDP-GlcNAc and UDP-GalNAc. Data were analyzed using the

589 MassLynx and TargetLynx software (Waters). 


\section{Acknowledgments}

591 We thank all M.S.D. laboratory members for lively and helpful discussions. We thank

592 the Metabolomics Core Facility and the Comparative Biology Facility at the Max Planck

593 Institute for Biology of Ageing. We thank Maribel Schönewolff, Emanuel Bruckisch,

594 and Klara Schilling for their support with mouse genotyping.

595 K.A. was supported by the Cologne Graduate School for Ageing Research. M.S.D.

596 was supported by ERC-StG 640254 and ERC-PoC 768524, by the Deutsche

597 Forschungsgemeinschaft (DFG, German Research Foundation) - Projektnummer

59873111208 - SFB 829, and by the Max Planck Society.

599 Conflict of Interest statement

600 The authors declare no potential conflict of interests.

\section{Authors' contributions}

602 K.A, M.D.H., A.M., and M.S.D. designed the research. K.A. and M.D.H. wrote the 603 manuscript. M.P. and A.M. performed mouse phenotyping. K.A. performed all other 604 experiments. 
606

607

608

609

610

611

612

613

614

615

616

617

618

619

620

621

622

623

624

625

626

627

628

629

630

631

632

633

634

635

636

637

638

639

640

641

642

Baron, Alain D. et al. 1995. "Glucosamine Induces Insulin Resistance in Vivo by Affecting GLUT 4 Translocation in Skeletal Muscle: Implications for Glucose Toxicity." Journal of Clinical Investigation 96(6): 2792-2801.

Denisot, Marie Ange, François Le Goffic, and Bernard Badet. 1991. "Glucosamine-6Phosphate Synthase from Escherichia Coli Yields Two Proteins upon Limited Proteolysis: Identification of the Glutamine Amidohydrolase and 2R Ketose/Aldose Isomerase-Bearing Domains Based on Their Biochemical Properties." Archives of Biochemistry and Biophysics 288(1): 225-30.

Denzel, Martin S. et al. 2014. "Hexosamine Pathway Metabolites Enhance Protein Quality Control and Prolong Life." Cell.

Denzel, Martin S., and Adam Antebi. 2015. "Hexosamine Pathway and (ER) Protein Quality Control." Current Opinion in Cell Biology 33(Figure 1): 14-18. http://dx.doi.org/10.1016/j.ceb.2014.10.001.

Einstein, Francine H. et al. 2008. "Enhanced Activation of a 'Nutrient-sensing' Pathway with Age Contributes to Insulin Resistance." The FASEB Journal.

Fardini, Yann, Vanessa Dehennaut, Tony Lefebvre, and Tarik Issad. 2013. "OGlcNAcylation: A New Cancer Hallmark?" Frontiers in Endocrinology 4(AUG): 114.

Han, Dong Ho, May M. Chen, and John O. Holloszy. 2003. "Glucosamine and Glucose Induce Insulin Resistance by Different Mechanisms in Rat Skeletal Muscle." American Journal of Physiology - Endocrinology and Metabolism.

Hanisch, F. G. 2001. "O-Glycosylation of the Mucin Type." Biological Chemistry.

Hart, Gerald W. 1997. "Dynamic O-Linked Glycosylation of Nuclear and Cytoskeletal Proteins." Annual Review of Biochemistry.

Hebert, Leon F. et al. 1996. "Overexpression of Glutamine:Fructose-6-Phosphate Amidotransferase in Transgenic Mice Leads to Insulin Resistance." Journal of Clinical Investigation.

Horn, Moritz et al. 2020. "Hexosamine Pathway Activation Improves Protein Homeostasis through the Integrated Stress Response." iScience 23(3): 100887. https://doi.org/10.1016/j.isci.2020.100887.

Kline, Christina Leah B., Tabitha L. Schrufer, Leonard S. Jefferson, and Scot R. Kimball. 2006. "Glucosamine-Induced Phosphorylation of the a-Subunit of Eukaryotic Initiation Factor 2 Is Mediated by the Protein Kinase R-like Endoplasmic-Reticulum Associated Kinase." International Journal of Biochemistry and Cell Biology 38(5-6): 1004-14.

Lemberg, Kathryn M., James J. Vornov, Rana Rais, and Barbara S. Slusher. 2018. “We're Not 'Don' yet: Optimal Dosing and Prodrug Delivery of 6-Diazo-5-Oxo-L- 
Norleucine." Molecular Cancer Therapeutics 17(9): 1824-32.

644

645

646

647

648

649

650

651

652

653

654

655

656

657

658

659

660

661

662

663

664

665

666

667

668

669

670

671

672

673

674

675

676

677

678

679

680

681

682

Li, Lili et al. 2017. "High Expression of GFAT1 Predicts Unfavorable Prognosis in Patients with Hepatocellular Carcinoma." Oncotarget 8(12): 19205-17.

Lombardi, Angela et al. 2012. "Increased Hexosamine Biosynthetic Pathway Flux Dedifferentiates INS-1E Cells and Murine Islets by an Extracellular SignalRegulated Kinase (ERK)1/2-Mediated Signal Transmission Pathway." Diabetologia 55(1): 141-53.

Marshall, S., V. Bacote, and R. R. Traxinger. 1991. "Discovery of a Metabolic Pathway Mediating Glucose-Induced Desensitization of the Glucose Transport System: Role of Hexosamine in the Induction of Insulin Resistance." Journal of Biological Chemistry.

McClain, Donald A. et al. 2002. "Altered Glycan-Dependent Signaling Induces Insulin Resistance and Hyperleptinemia." Proceedings of the National Academy of Sciences of the United States of America.

Olchowy, Jarosław, Krzysztof Kur, Paweł Sachadyn, and Sławomir Milewski. 2006. "Construction, Purification, and Functional Characterization of His-Tagged Candida Albicans Glucosamine-6-Phosphate Synthase Expressed in Escherichia Coli." Protein Expression and Purification.

Pan, Simon et al. 2020. "Preservation of a Remote Fear Memory Requires New Myelin Formation." Nature Neuroscience.

Parodi, Armando J. 2000. "Role of N-Oligosaccharide Endoplasmic Reticulum Processing Reactions in Glycoprotein Folding and Degradation." Biochemical Journal.

Rossetti, Luciano et al. 1995. "In Vivo Glucosamine Infusion Induces Insulin Resistance in Normoglycemic but Not in Hyperglycemic Conscious Rats." Journal of Clinical Investigation.

Ruegenberg, Sabine et al. 2020. "Loss of GFAT-1 Feedback Regulation Activates the Hexosamine Pathway That Modulates Protein Homeostasis." Nature Communications 11(1): 687. https://doi.org/10.1038/s41467-020-14524-5.

Ruegenberg, Sabine et al. 2021. "Protein Kinase A Controls the Hexosamine Pathway by Tuning the Feedback Inhibition of GFAT-1." Nature Communications.

Ryczko, Michael C. et al. 2016. "Metabolic Reprogramming by Hexosamine Biosynthetic and Golgi N-Glycan Branching Pathways." Scientific Reports 6(November 2015): 1-15. http://dx.doi.org/10.1038/srep23043.

Schwenk, Frieder, Udo Baron, and Klaus Rajewsky. 1995. "A Cre -Transgenic Mouse Strain for the Ubiquitous Deletion of LoxP -Flanked Gene Segments Including Deletion in Germ Cells ." Nucleic Acids Research 23(24): 5080-81. https://doi.org/10.1093/nar/23.24.5080.

Senderek, Jan et al. 2011. "Hexosamine Biosynthetic Pathway Mutations Cause Neuromuscular Transmission Defect." American Journal of Human Genetics. 
683

684

685

686

687

688

689

690

691

692

693

694

695

696

697

698

699

700

701

702

703

704

705

706

707

708

709

710

711

712

713

714

715

716

717

Shintani, Tomoya, Yuhei Kosuge, and Hisashi Ashida. 2018. "Glucosamine Extends the Lifespan of Caenorhabditis Elegans via Autophagy Induction Glucosamine Extends Nematode Lifespan via Autophagy Induction." Journal of Applied Glycoscience 65(3): 37-43.

Silverman, Jerome L. 1963. "Glucosamine Inhibition of [1-14C]Glucose Oxidation as Measured by Rat Adipose Tissue in Vitro." BBA - Biochimica et Biophysica Acta.

Steadman, Patrick E. et al. 2020. "Disruption of Oligodendrogenesis Impairs Memory Consolidation in Adult Mice." Neuron.

Stocchi, V. et al. 1981. "Rabbit Red Blood Cell Hexokinase. Evidence for Two Distinct Forms, and Their Purification and Characterization from Reticulocytes." Journal of Biological Chemistry.

Sy, Michael et al. 2020. "N-Acetylglucosamine Drives Myelination by Triggering Oligodendrocyte Precursor Cell Differentiation." Journal of Biological Chemistry 295(51): 17413-24. http://dx.doi.org/10.1074/jbc.RA120.015595.

Takahashi, Miwa et al. 2009. "Lack of Chronic Toxicity or Carcinogenicity of Dietary N-Acetylglucosamine in F344 Rats." Food and Chemical Toxicology.

Veerababu, Geddati et al. 2000. "Overexpression of Glutamine:Fructose-6-Phosphate Amidotransferase in the Liver of Transgenic Mice Results in Enhanced Glycogen Storage, Hyperlipidemia, Obesity, and Impaired Glucose Tolerance." Diabetes.

Virkamaki, A. 1997. "Activation of the Hexosamine Pathway by Glucosamine in Vivo Induces Insulin Resistance in Multiple Insulin Sensitive Tissues." Endocrinology.

Vorhees, Charles V., and Michael T. Williams. 2006. "Morris Water Maze: Procedures for Assessing Spatial and Related Forms of Learning and Memory." Nature Protocols.

Weihofen, Wilhelm A. et al. 2006. "Structures of Human N-Acetylglucosamine Kinase in Two Complexes with N-Acetylglucosamine and with ADP/Glucose: Insights into Substrate Specificity and Regulation." Journal of Molecular Biology.

Weimer, Sandra et al. 2014. "D-Glucosamine Supplementation Extends Life Span of Nematodes and of Ageing Mice." Nature communications 5: 3563.

Wells, L., K. Vosseller, and G. W. Hart. 2003. "A Role for N-Acetylglucosamine as a Nutrient Sensor and Mediator of Insulin Resistance." Cellular and Molecular Life Sciences.

Wheatley, Elizabeth G. et al. 2019. "Neuronal O-GlcNAcylation Improves Cognitive Function in the Aged Mouse Brain." Current Biology. 
Figures

A

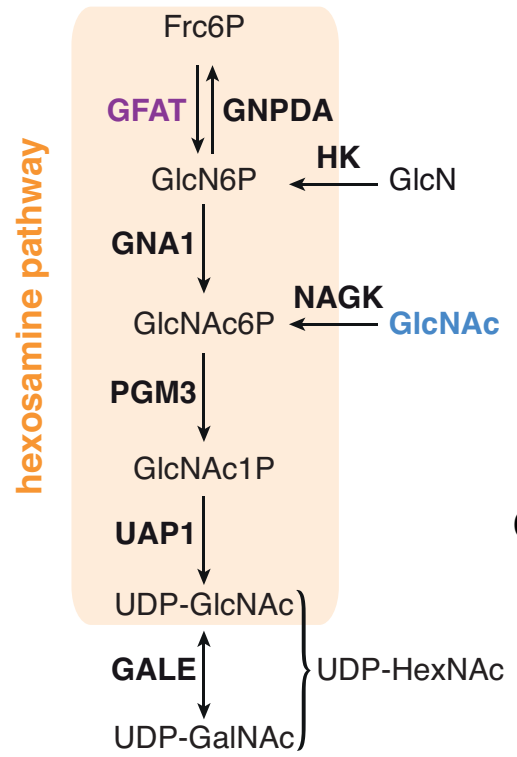

E $\rightarrow$ control $\rightarrow 1 \%$ GlcNAc
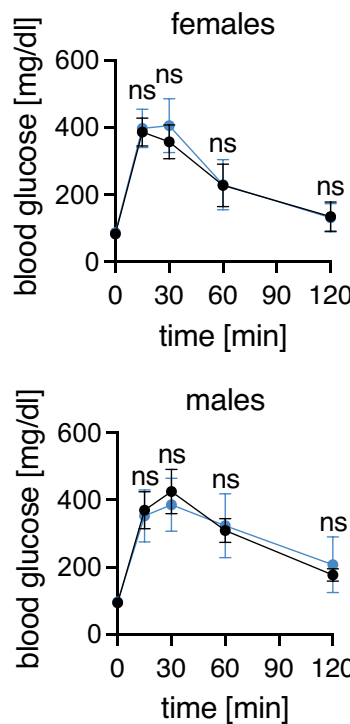

G

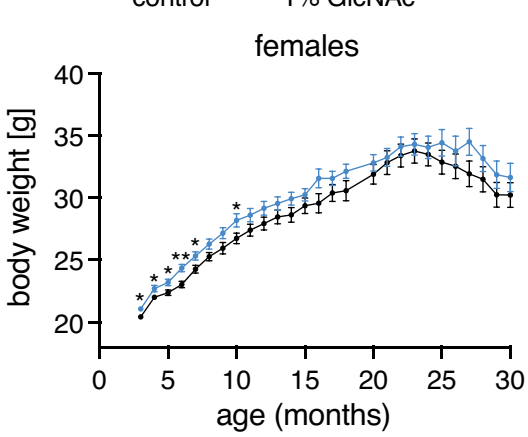

C

$\mathbf{F}$
B $\square$ control $\square 1 \%$ GlcNAc
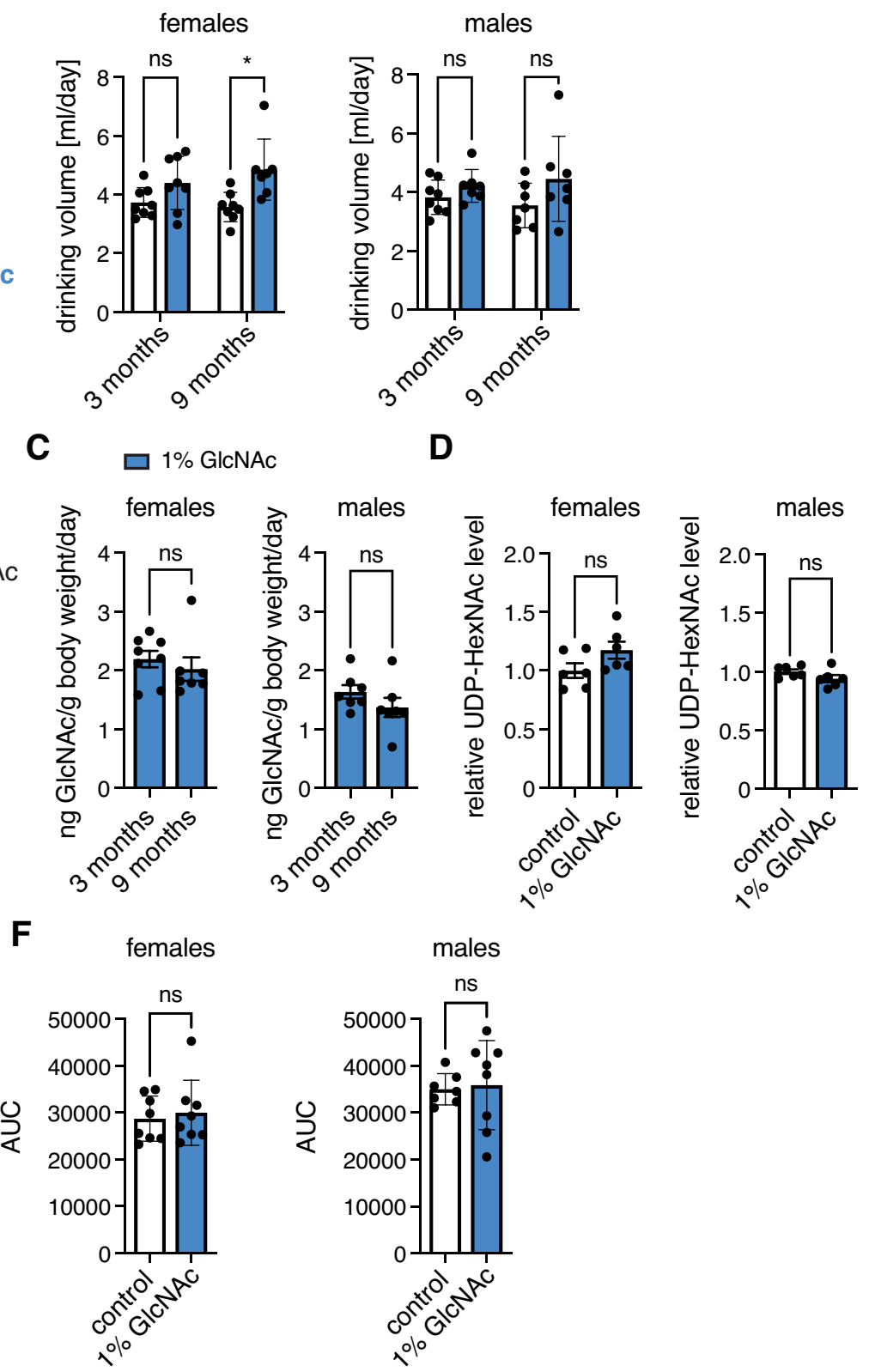

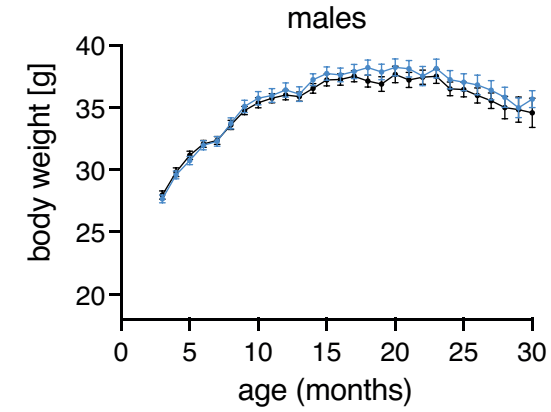

Figure 1: Hexosamine biosynthetic pathway activation by GIcNAc feeding does 
722 biosynthetic pathway. The rate-limiting enzyme GFAT is depicted in purple, GlcNAc is

723 marked in blue. (B) Drinking volume of control (white) and GlcNAc-treated mice (blue)

724 of both sexes at 3 and 9 months of age. Data are presented as mean $\pm S D(n \geq 7)$.

725 Two-way ANOVA, Tukey's post-test; * $p<0.05$; ns: not significant. (C) GlcNAc

726 consumption in $\mathrm{ng} / \mathrm{g}$ body weight per day of mice of both sexes at 3 and 9 months of

727 age. Data are presented as mean \pm SD $(n \geq 7)$. Unpaired t-test; ns: not significant. (D)

728 Relative UDP-HexNAc levels in hemibrains of control (white) and GlcNAc-treated mice

729 (blue) of both sexes. Data are presented as mean \pm SEM ( $n=6)$. Unpaired t-test; $n s$ :

730 not significant. (E) Blood glucose concentration at 0 (fasting), 15, 30, 60, and 120 min

731 after intraperitoneal injection of glucose solution ( $2 \mathrm{~g} / \mathrm{kg}$ body weight) of control (black)

732 and GlcNAc-treated mice (blue) of both sexes at 10 months of age. Data are presented

733 as mean $\pm S D(n \geq 7)$. Multiple unpaired t-tests; ns: not significant $(F)$ Area under the

734 curve (AUC) calculated using data shown in (E). Data are presented as mean \pm SD

735 ( $\mathrm{n} \geq 7$ ). Unpaired t-test; ns: not significant $(\mathrm{G})$ Body weight of control (black) and

736 GlcNAc-treated mice (blue) of both sexes from 3 to 30 months of age. Data are

737 presented as mean $\pm S D(n \geq 13)$. Multiple unpaired t-tests; ${ }^{* *} p<0.01$; ${ }^{*} p<0.05$; only

738 significant changes are indicated. 
A
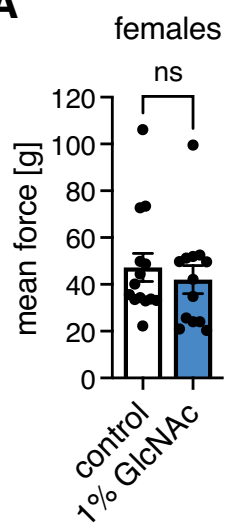

C

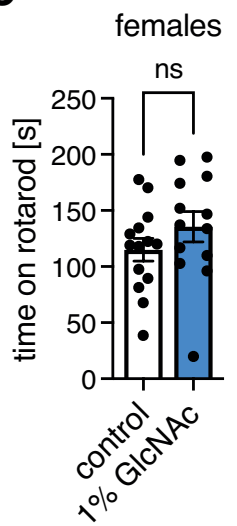

$\mathbf{E}$
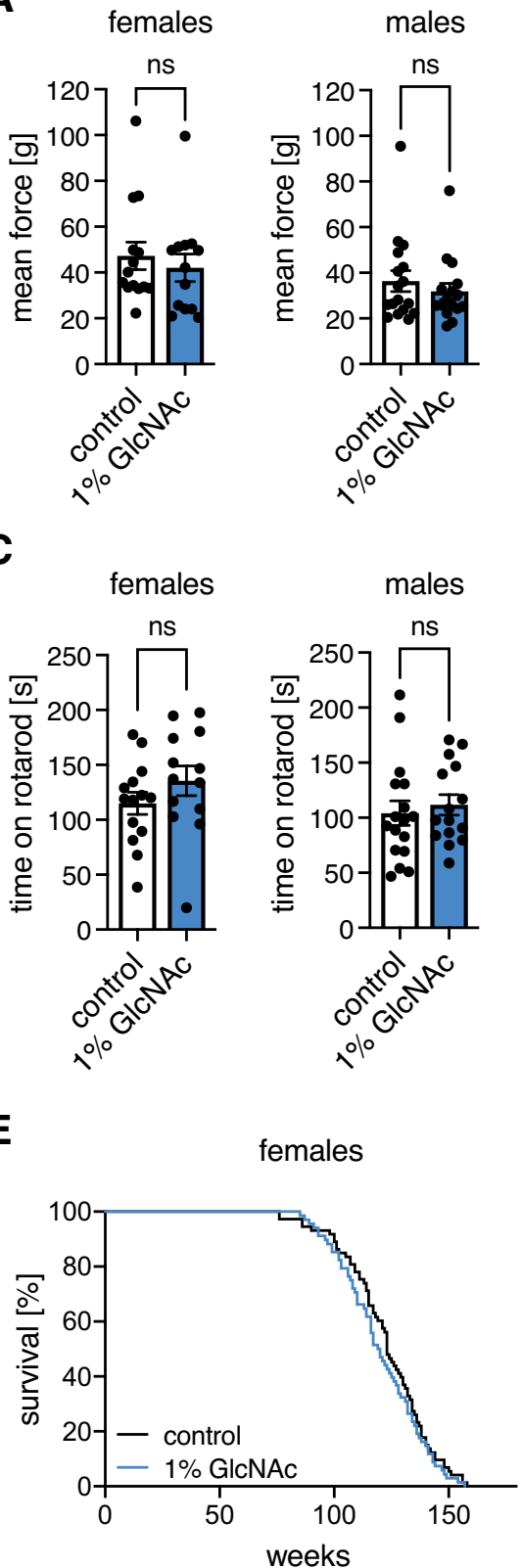

B
D
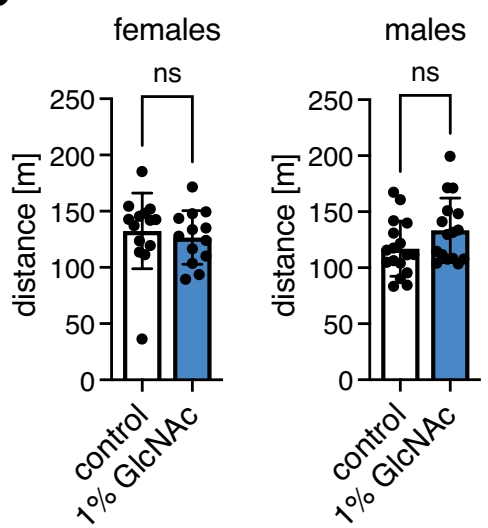

males
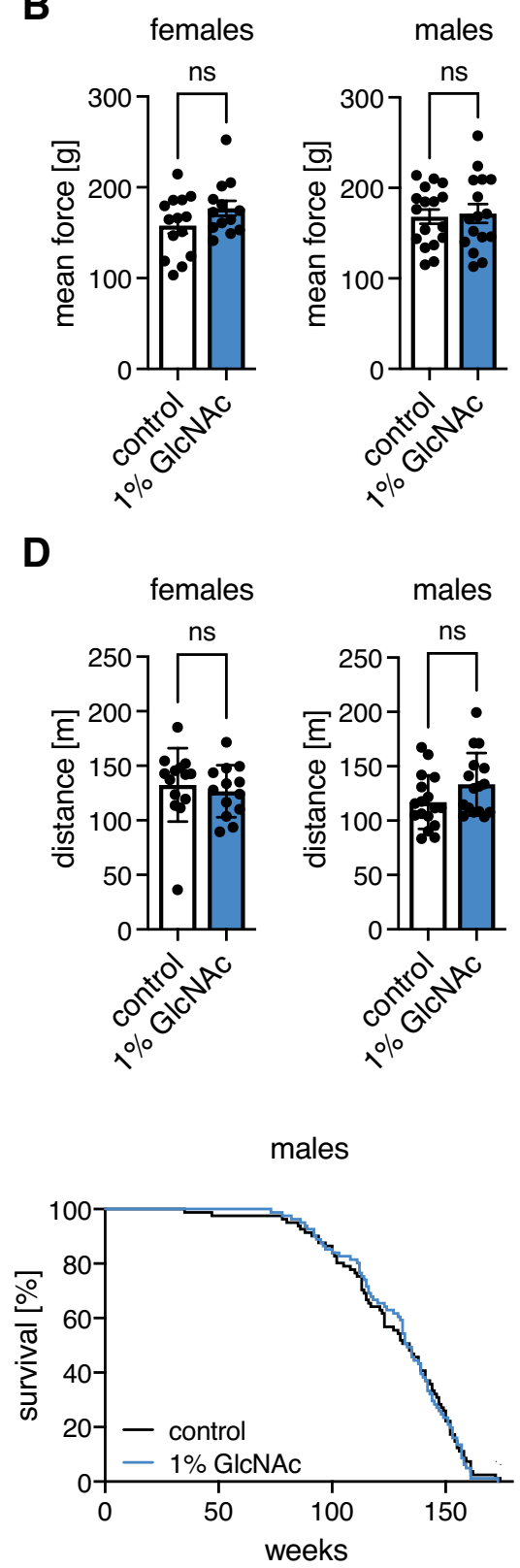

740 Figure 2: GIcNAc supplementation does not influence fitness of mice. (A) Mean

741 force measured in a grip strength test with two paws of control (white) and GlcNAc-

742 treated mice (blue) of both sexes at 6 months of age. (B) Mean force measured in a 743 grip strength test with four paws of control (white) and GlcNAc-treated mice (blue) of 744 both sexes at 6 months of age. (C) Maximal time on a rotarod of control (white) and 745 GlcNAc-treated mice (blue) of both sexes at 6 months of age. (A-C) Data are 746 presented as mean \pm SEM $(n \geq 13)$. (D) Maximal distance on a treadmill of control 747 (white) and GlcNAc-treated mice (blue) of both sexes from at 6 months of age. Data 748 are presented as mean \pm SD $(n \geq 13)$. (A-D) Unpaired t-test; ns: not significant $(E)$ 749 Lifespan analysis of control (black) and GlcNAc-treated mice (blue) of both sexes 750 (females: $n=69$; males: $n=82$ ). 
A

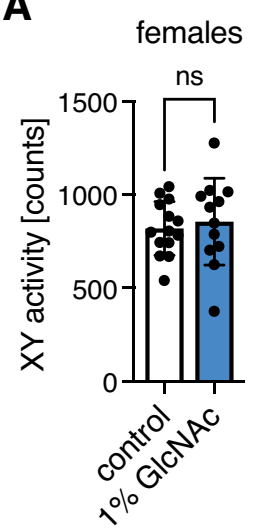

C

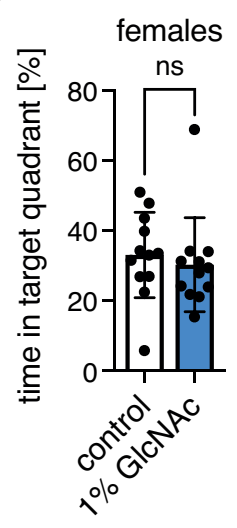

E

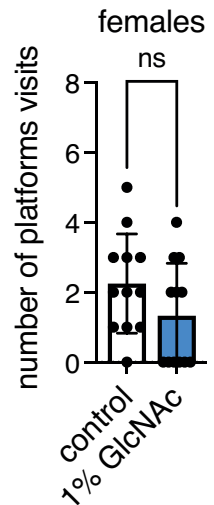

B
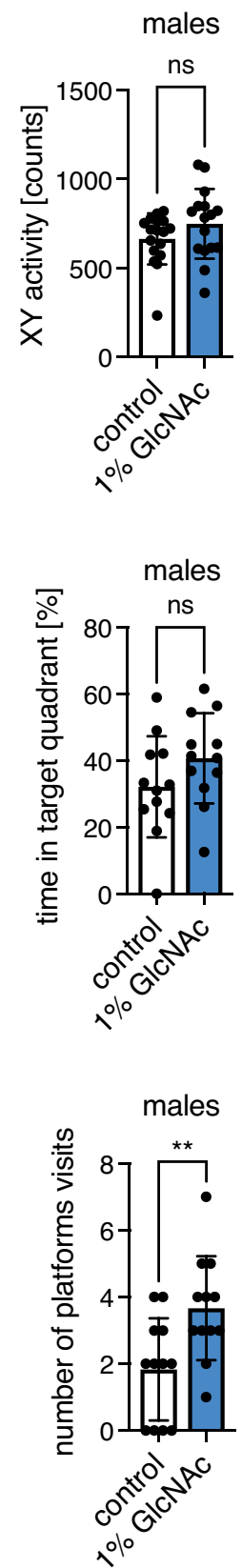

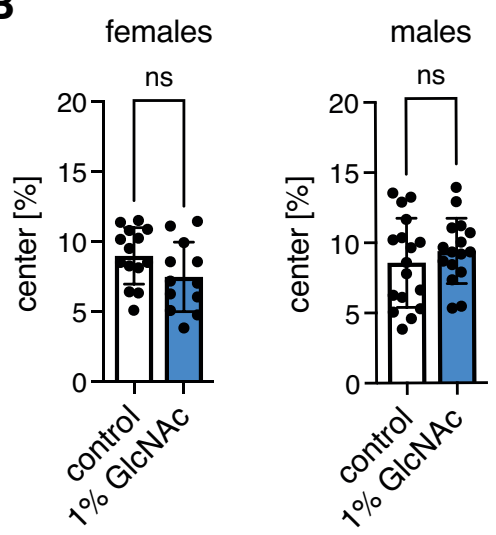

D

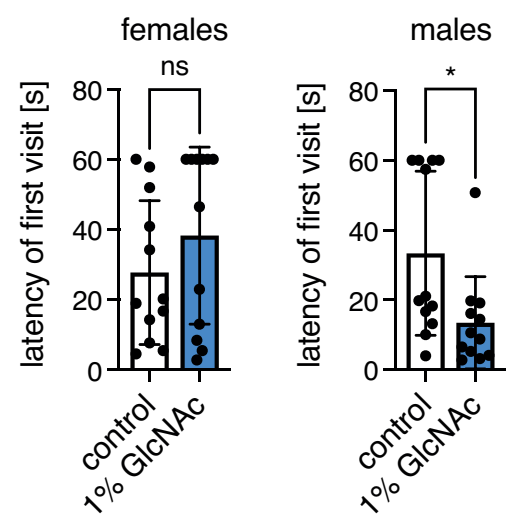

752 Figure 3: GIcNAc feeding improves memory of young male mice. (A) XY activity

753 measured in the open field test of control (white) and GlcNAc-treated mice (blue) of 754 both sexes at 6 months of age. (B) Percent of distance spent in the center of the open 755 field of control (white) and GlcNAc-treated mice (blue) of both sexes at 6 months of age. (A-B) Data are presented as mean $\pm S D(n \geq 12)$. (C) Percent of time spend in the target quadrant, (D) Latency of the first platform visit, and $(E)$ Number of platform visits upon removal of the hidden platform in the Morris water maze test of control (white) and GlcNAc-treated mice (blue) of both sexes at 4 months of age. (C-E) Data are presented as mean $\pm S D(n=12)$. (A-E) Unpaired t-test; ${ }^{* *} p<0.01$; * $p<0.05$; ns: not significant. 
A

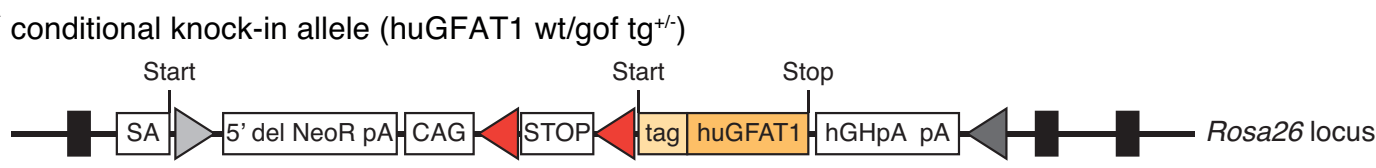

constitutive knock-in allele (huGFAT1 wt/gof OE)

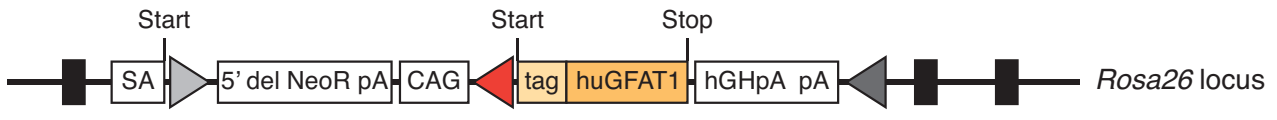

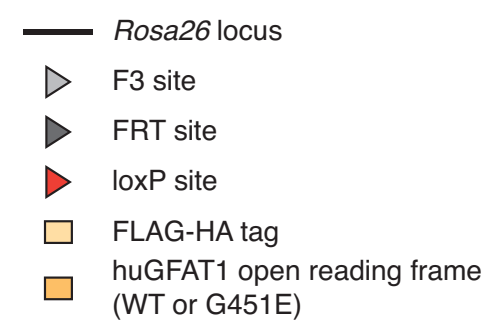

B

E

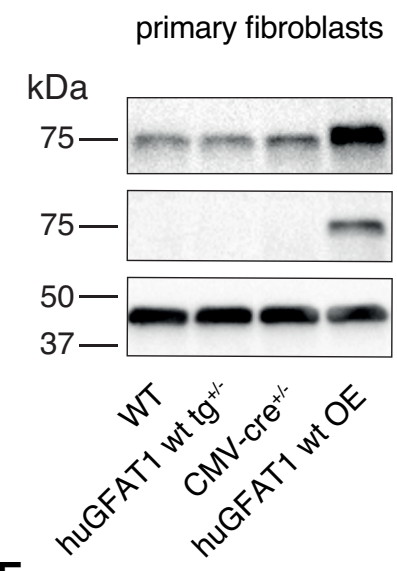

females

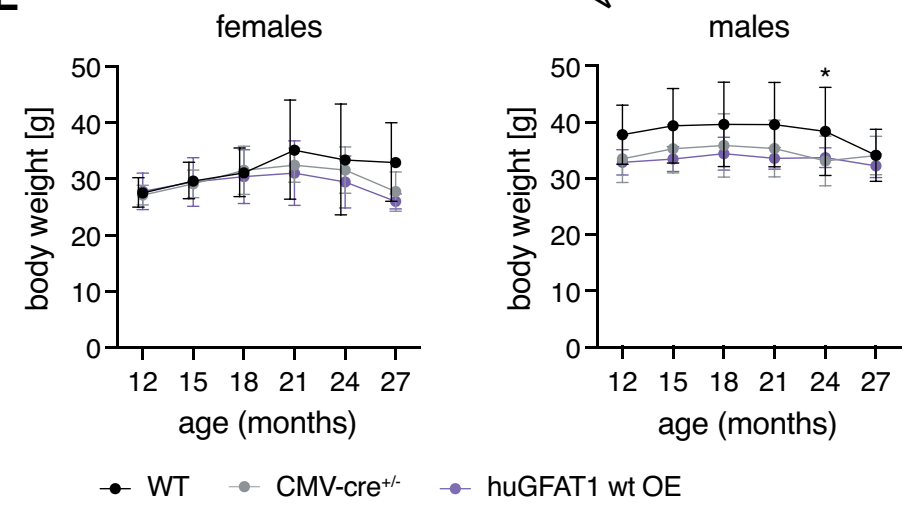

splice acceptor site

5' del NeoR pA neomycin resistance cassette

CAG CAG promoter cassette

STOP transcription termination cassette

hGHpA pA and an additional polyadenylation signal
D
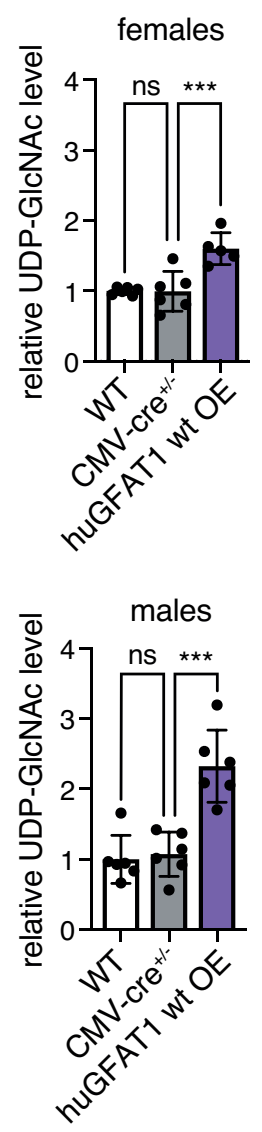

C
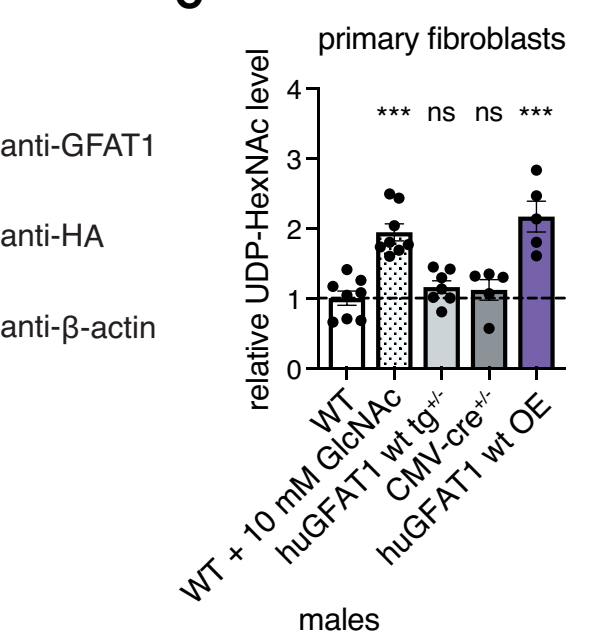

763

Figure 4: HBP activation by huGFAT1 wt OE does not influence body weight in mice. (A) Schematic representation of the transgene. The expression cassette was inserted in the Rosa26 locus (conditional knock-in allele). Upon cre-mediated deletion of the transcription termination cassette, FLAG-HA tagged human GFAT1 is expressed under the control of the chicken $\beta$-actin promoter (constitutive knock-in allele). (B) Western blot analysis of GFAT1 and HA expression in primary fibroblasts 
769 isolated from newborn mice $(n=1)$. $\beta$-actin was used as loading control. (C) Relative

770 UDP-HexNAc levels in primary fibroblasts. (D) Relative UDP-GlcNAc levels in

771 hemibrain isolated from 3 months old control and huGFAT1 wt OE mice of both sexes.

772 (C-D) Data are presented as mean \pm SEM $(n \geq 5)$. One-way ANOVA, Dunnett's post773 test; ${ }^{* * *} p<0.001$; ns: not significant. (E) Body weight of control and huGFAT1 wt OE

774 mice of both sexes from 12 to 27 months of age. Data are presented as mean \pm SD

$775(\mathrm{n} \geq 2)$. Two-way ANOVA, Dunnett's post-test. Statistical significance was calculated

776 compared to $\mathrm{CMV}_{-\mathrm{cre}^{+/}}$mice at each time point; only significant changes are

777 indicated. * $p<0.05$ 
A

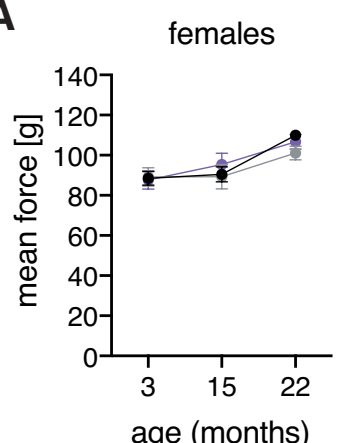

$\rightarrow$ WT $\rightarrow$ CMV-cre ${ }^{+1}$

C

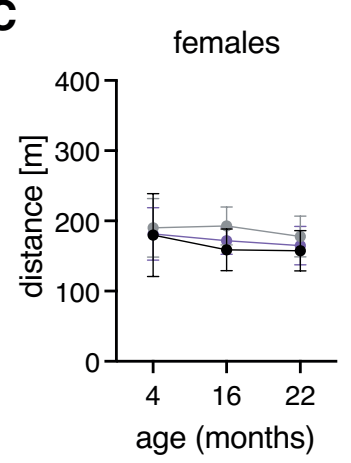

$\rightarrow$ WT $\rightarrow$ CMV-cre ${ }^{+-} \rightarrow$ huGFAT1 wt OE
B

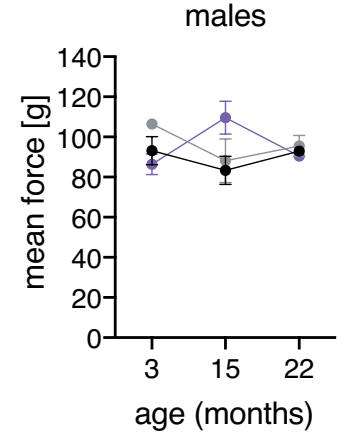

- hugFaT1 wt OE

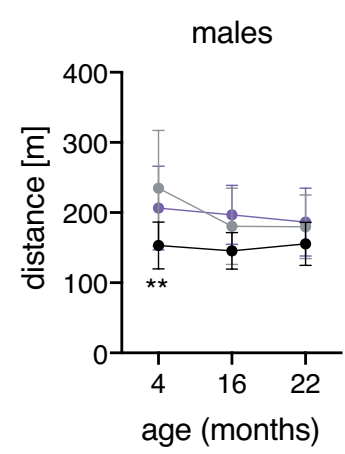

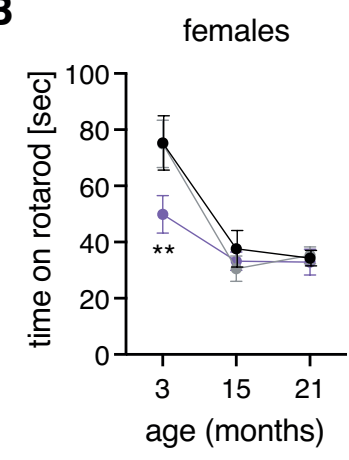

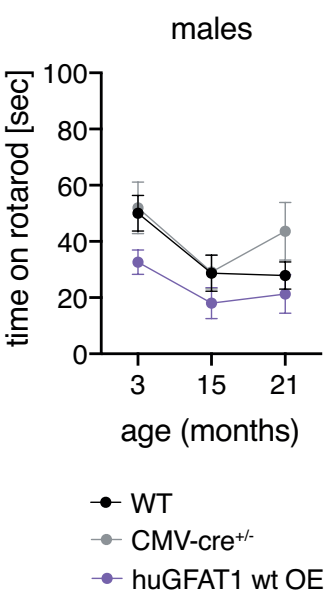

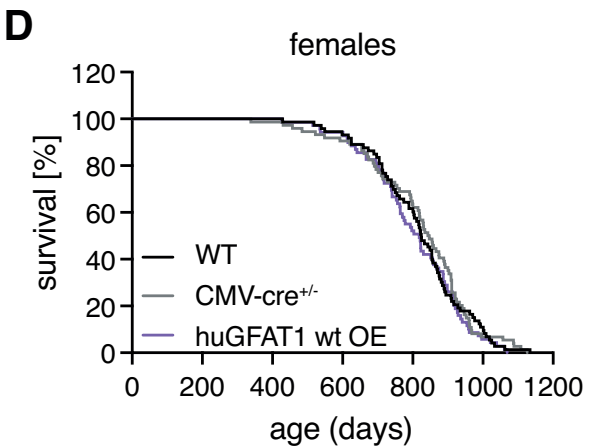

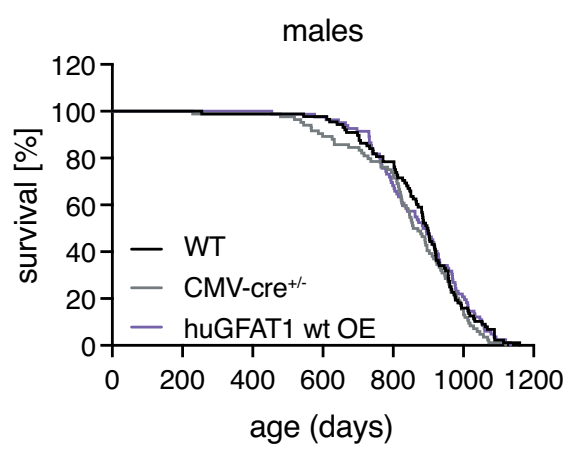

779 Figure 5: HBP activation by huGFAT1 wt OE does not affect fitness of mice. (A) 780 Mean force measured in a grip strength test with two paws of control and huGFAT1 wt 781 OE mice of both sexes at 3, 15 and 22 months of age. (B) Maximal time on a rotarod 782 of control and huGFAT1 wt OE mice of both sexes at 3, 15 and 21 months of age. 783 (A-B) Data are presented as mean \pm SEM $(n \geq 2)$. (C) Maximal distance on a treadmill 784 of control and huGFAT1 wt OE mice of both sexes at 4, 16 and 22 months of age. 785 Data are presented as mean \pm SD $(n \geq 4)$. (A-C) Two-way ANOVA, Dunnett's post-test. 786 Statistical significance was calculated compared to CMV-cre ${ }^{+/-}$mice at each time point; 787 only significant changes are indicated. ${ }^{* *} p<0.01$ (D) Lifespan analysis of control and 788 huGFAT1 wt OE mice of both sexes (females: $n \geq 68$; males: $n \geq 80$ ). Survival of WT and 789 CMV-cre ${ }^{+/-}$mice is also shown in Figure S4b. 
A

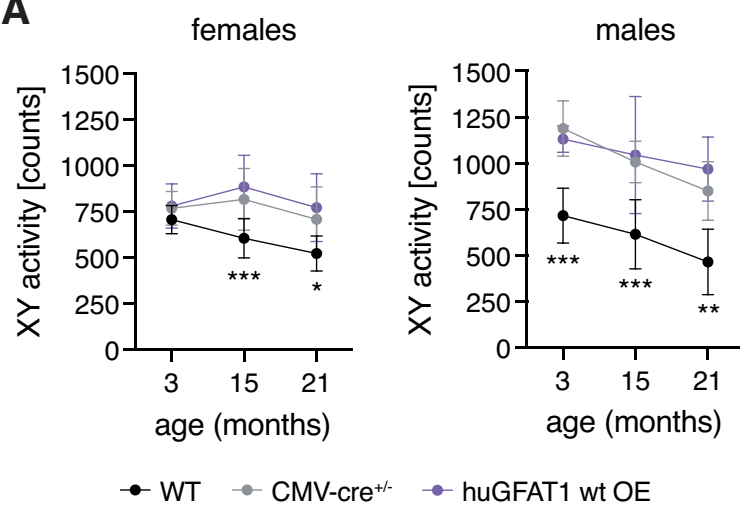

C
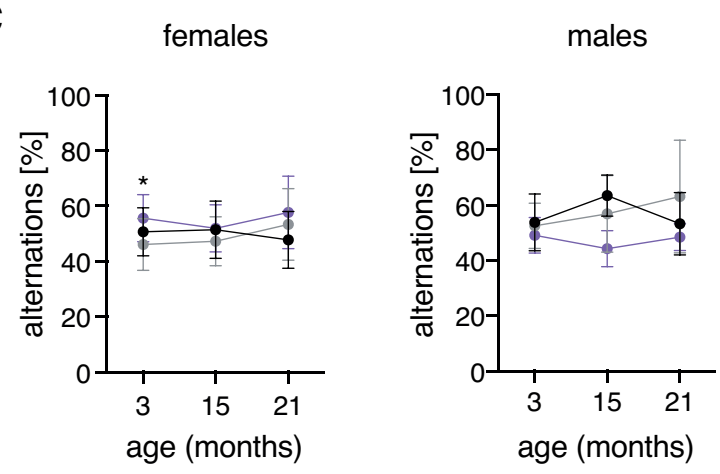

$\bullet$ WT $\rightarrow \mathrm{CMV}^{-\mathrm{cre}^{+/}} \rightarrow$ huGFAT1 wt OE

E

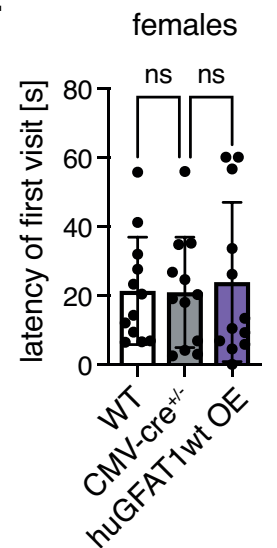

B
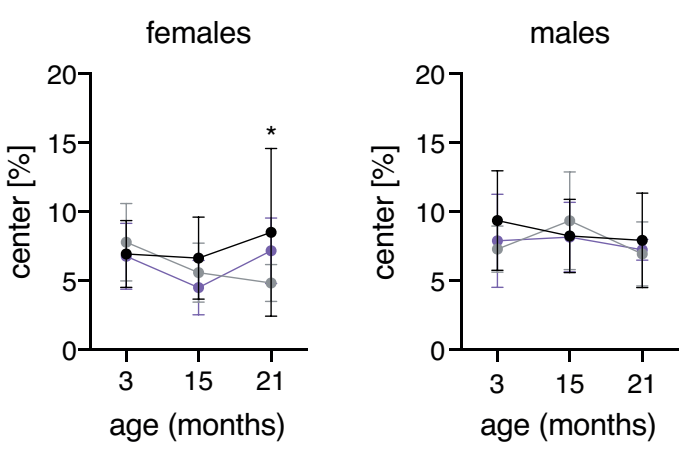

D
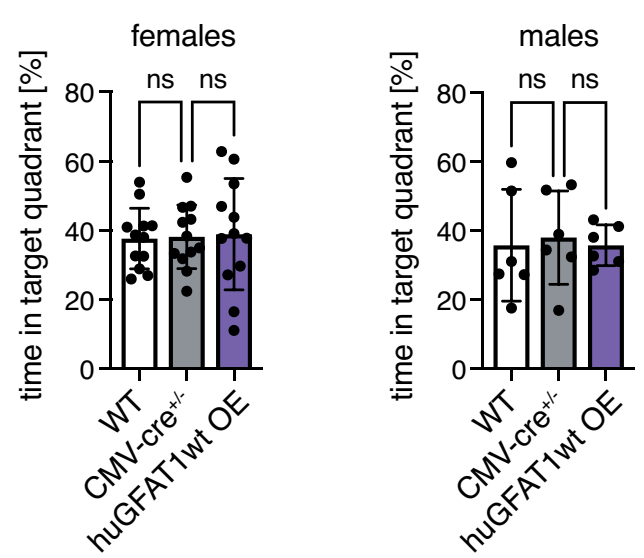

$\mathbf{F}$

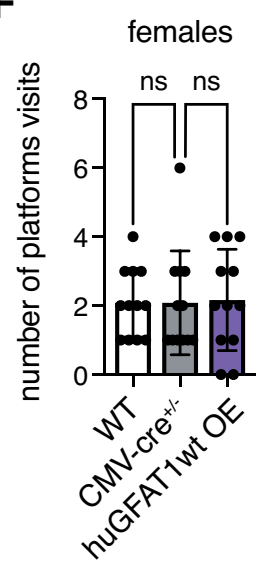

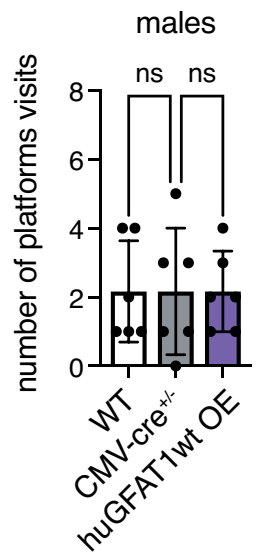

Figure 6: HBP activation by huGFAT1 wt OE does not influence spontaneous locomotor and exploratory behavior or memory of mice. (A) XY activity measured in the open field test of control and huGFAT1 wt OE mice of both sexes at 3, 15 and 21 months of age. (B) Percent of distance spent in the center of the open field of control and huGFAT1 wt OE mice of both sexes at 3, 15 and 21 months of age. (C) Percent of alternations measured in the $Y$ maze test of control and huGFAT1 wt OE mice of both sexes at 3,15 and 21 months of age. (A-C) Data are presented as mean \pm SD $(n \geq 4)$. Two-way ANOVA, Dunnett's post-test. Statistical significance was calculated compared to $\mathrm{CMV}-\mathrm{cre}^{+/-}$mice at each time point; only significant changes are indicated. ${ }^{* * *} p<0.001 ;{ }^{* *} p<0.01 ;{ }^{*} p<0.05$ (D) Percent of time spend in the target 

wt OE mice of both sexes at 4 months of age. (D-F) Data are presented as mean $\pm S D$

804 ( $\mathrm{n} \geq 6)$. One-way ANOVA, Dunnett's post-test; ns: not significant. 
A

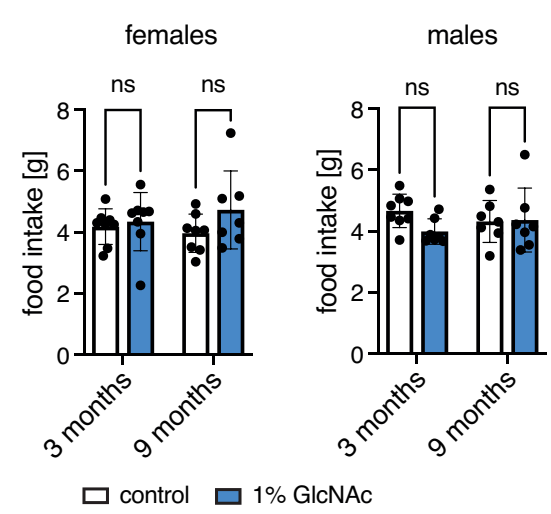

B

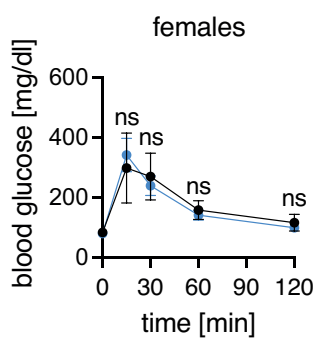

20 months

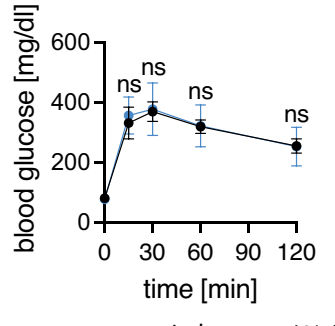

$\rightarrow$ control $\rightarrow 1 \%$ GlcNAc

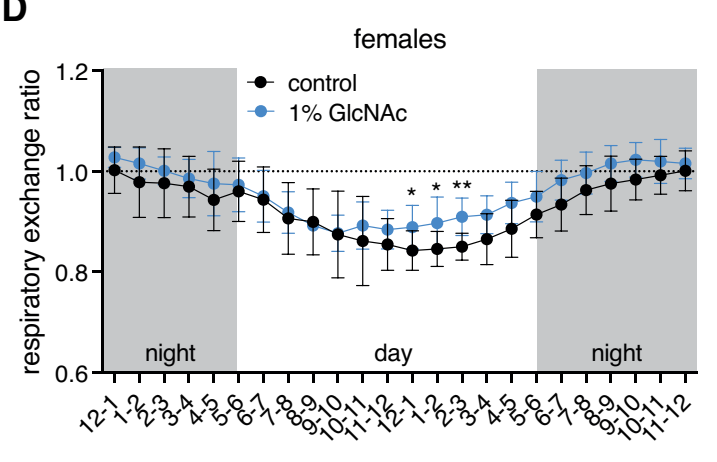

males

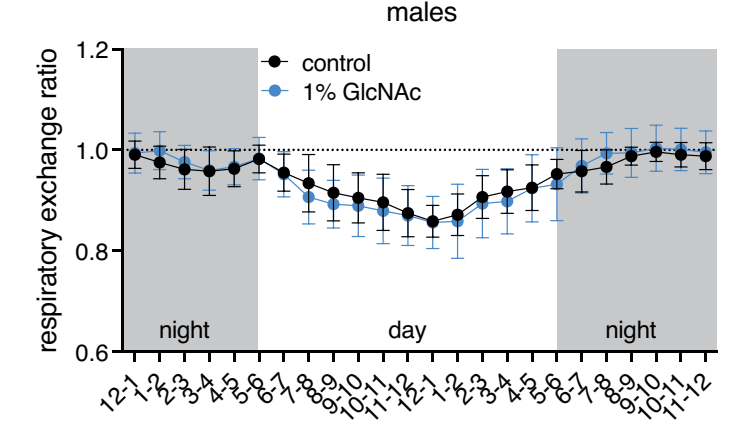

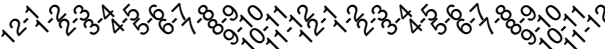
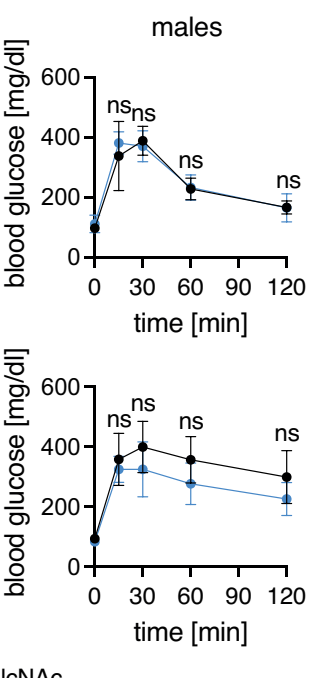

emales

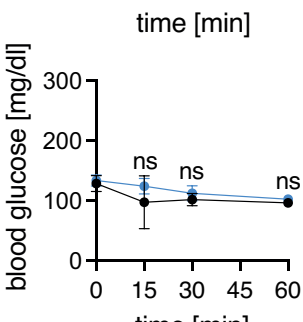

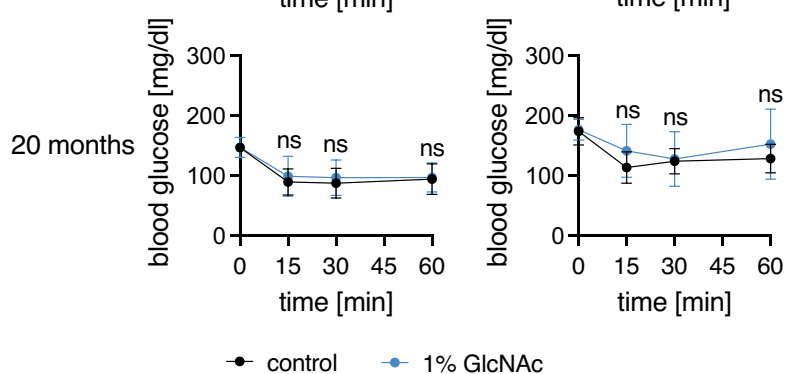

$\rightarrow$ control $\rightarrow 1 \%$ GlcNAc

Figure S1: GIcNAc supplementation does not influence food intake or insulin of both sexes at 3 and 9 months of age measured in the metabolic cages. Data are presented as mean $\pm S D(n \geq 7)$. Two-way ANOVA, Tukey's post-test; ns: not significant (B) Blood glucose concentration at 0 (fasting), 15, 30, 60, and 120 min after intraperitoneal injection of glucose solution ( $2 \mathrm{~g} / \mathrm{kg}$ body weight) of control (black) and GlcNAc-treated mice (blue) of both sexes at 4 and 20 months of age. (C) Blood glucose concentration before (0), and 15, 30, and 60 min after intraperitoneal injection 
817 ( $\mathrm{n} \geq 6$ ). Multiple unpaired t-tests; ${ }^{* *} \mathrm{p}<0.01$; * $\mathrm{p}<0.05$; ns: not significant (D) Respiratory 818 exchange ratio $\left(\mathrm{CO}_{2}\right.$ production $/ \mathrm{O}_{2}$ consumption) of control (black) and GlcNAc819 treated mice (blue) of both sexes during the day and at night (gray) at 9 months of age 820 measured in the metabolic cages. Data are presented as mean \pm SD $(n \geq 7)$. Multiple 821 unpaired t-tests; ${ }^{* *} p<0.01 ;{ }^{*} p<0.05$; only significant changes are indicated. 
A females

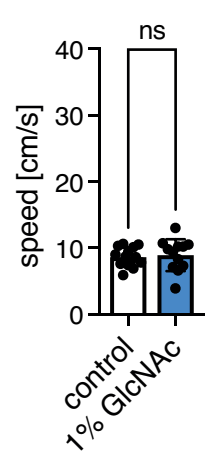

males

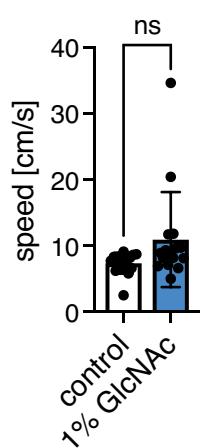

B

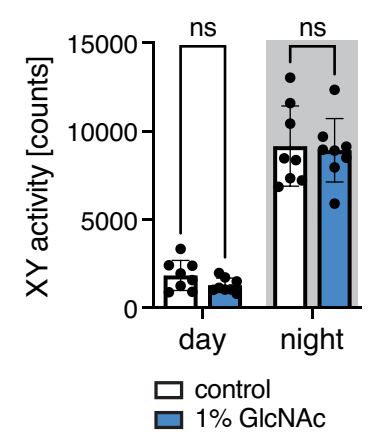

males

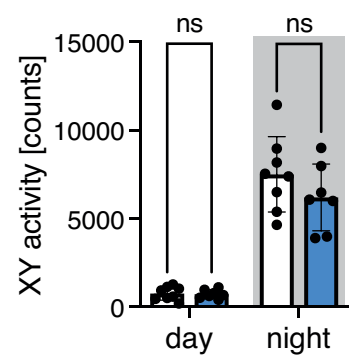

$c$

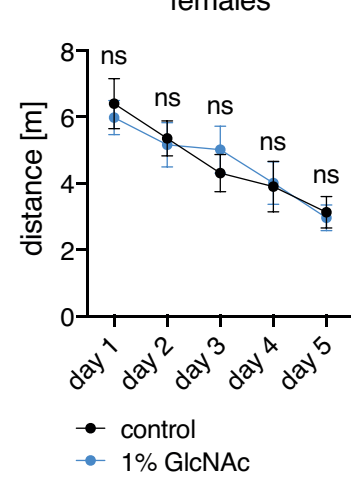

$\mathrm{E}$

females

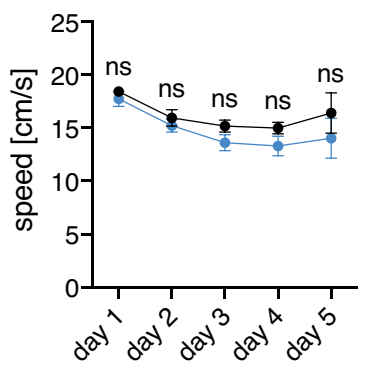

- control

- $1 \%$ GICNAc males

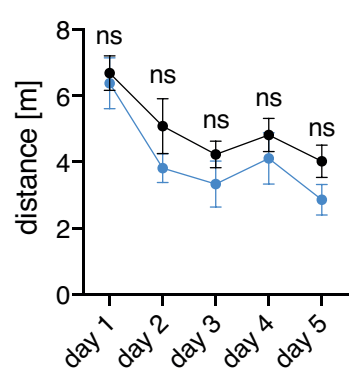

males

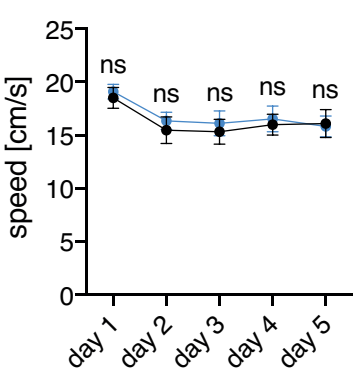

D

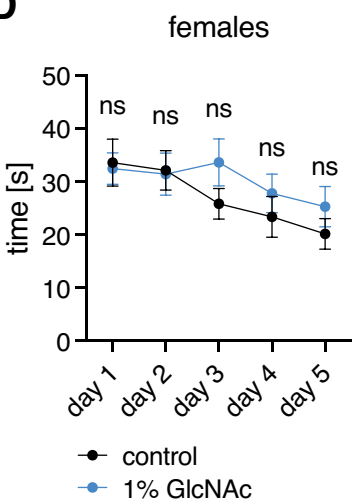

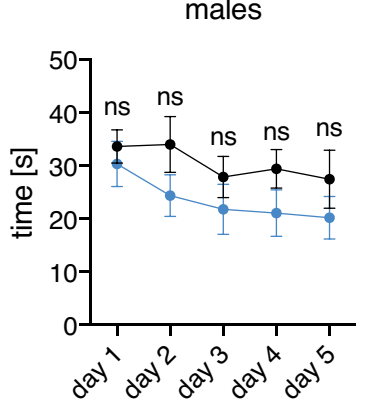

males

Figure S2: GIcNAc supplementation does not influence spontaneous locomotor

824 behavior or learning in mice. (A) Speed of control (white) and GlcNAc-treated mice 825 (blue) of both sexes at 6 months of age measured in the open field test. Data are 826 presented as mean $\pm S D(n \geq 12)$. Unpaired t-test; ns: not significant (B) XY activity of 827 control (white) and GlcNAc-treated mice (blue) of both sexes during the day and at 828 night (gray) at 3 months of age measured in the metabolic cages. Data are presented 829 as mean \pm SD $(n \geq 7)$. Two-way ANOVA, Tukey's post-test; ns: not significant. (C) 830 Distance and (D) Time until the mice reached the hidden platform, and (E) Swimming 831 speed of control (black) and GlcNAc-treated mice (blue) of both sexes at 4 months of 832 age during the Morris water maze training. (C-E) Data are presented as mean \pm SEM. 833 ( $n=12)$. The mean of four trials per day is plotted. Multiple unpaired t-tests; ns: not 834 significant. 
A

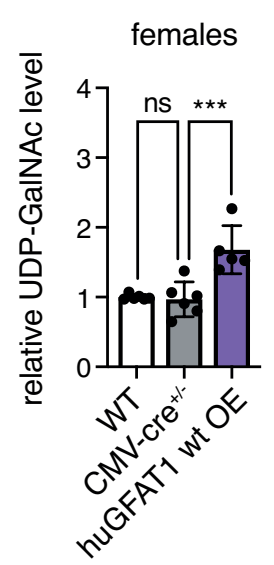

C
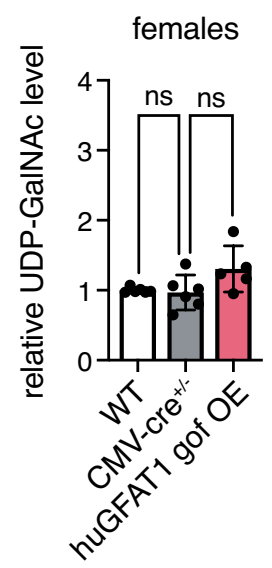

B
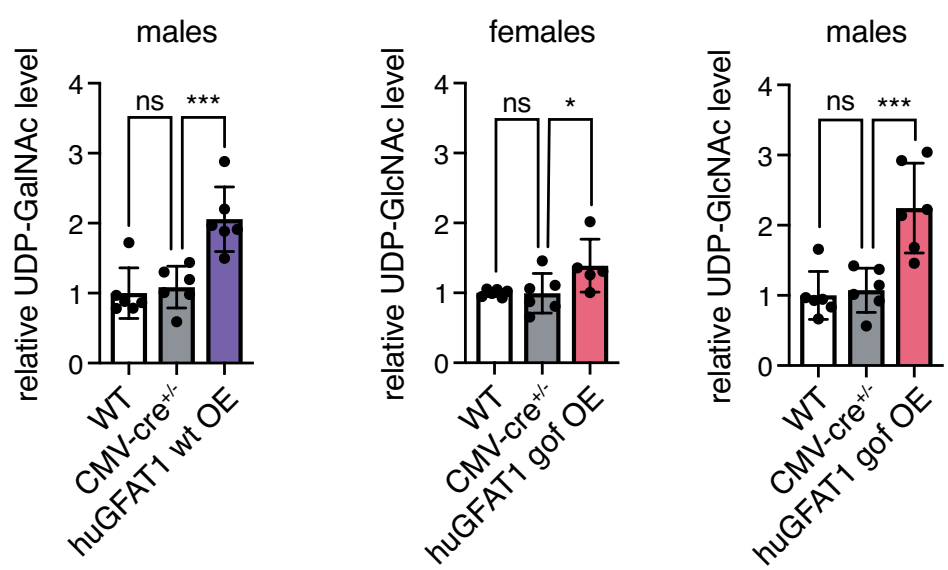

D

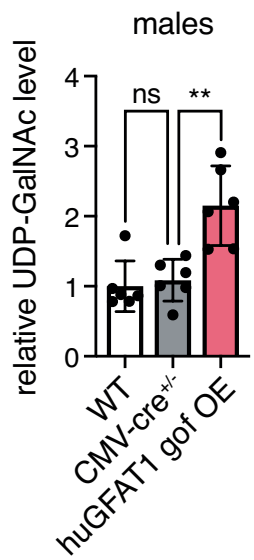

females

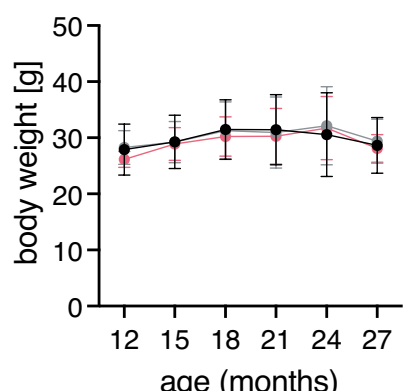

age (months)

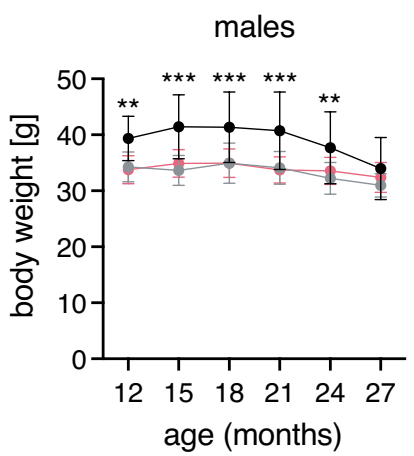

$\bullet$ WT $\rightarrow$ CMV-cre ${ }^{+/} \rightarrow$ huGFAT1 gof OE

Figure S3: HBP activation by huGFAT1 wt/gof OE in mice. (A) Relative

837 UDP-GalNAc levels in hemibrain isolated from 3 months old control and huGFAT1 wt 838 OE mice of both sexes. (B) Relative UDP-GIcNAc levels in hemibrain isolated from 8393 months old control and huGFAT1 gof OE mice of both sexes. (C) Relative 840 UDP-GalNAc levels in hemibrain isolated from 3 months old control and huGFAT1 gof 841 OE mice of both sexes. (A-C) Data are presented as mean \pm SEM. One-way ANOVA, 842 Dunnett's post-test; ${ }^{* * *} p<0.001$; ${ }^{* *} p<0.01$; ${ }^{*} p<0.05$; ns: not significant. (D) Body 843 weight of control and huGFAT1 gof OE mice of both sexes from 12 to 27 months of 844 age. Data are presented as mean $\pm S D(n \geq 5)$. Two-way ANOVA, Dunnett's post-test. 845 Statistical significance was calculated compared to CMV-cre ${ }^{+/-}$mice at each time point; only significant changes are indicated. ${ }^{* *} p<0.001 ;{ }^{* *} p<0.01$ 
A

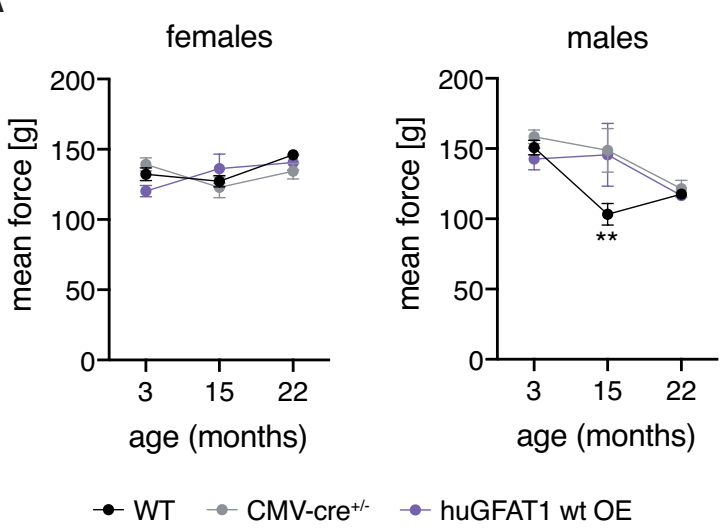

B

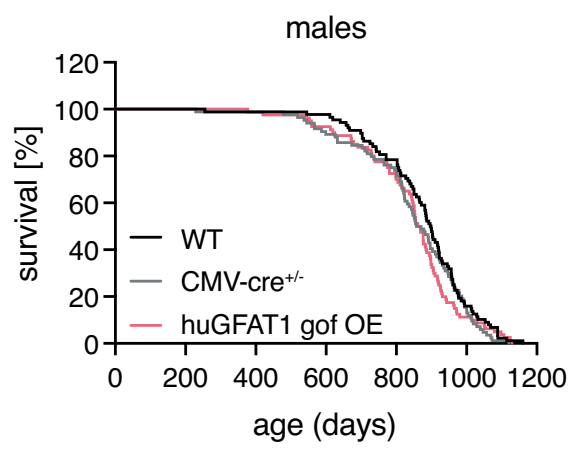

848 Figure S4: Genetic HBP activation does not affect fitness of mice. (A) Mean force 849 measured in a grip strength test with four paws of control and huGFAT1 wt OE mice 850 of both sexes at 3,15 and 22 months of age. Data are presented as mean $\pm S D(n \geq 4)$. 851 Two-way ANOVA, Dunnett's post-test. Statistical significance was calculated 852 compared to $\mathrm{CMV}-\mathrm{cre}^{+/-}$mice at each time point; only significant changes are 853 indicated. ${ }^{* *} p<0.01$ (B) Lifespan analysis of control and huGFAT1 gof OE mice of both 854 sexes (females: $n \geq 68$; males: $n \geq 80$ ). Survival of $W T$ and $C M V-$ cre $^{+/-}$mice is also 855 shown in Figure $5 d$. 
A

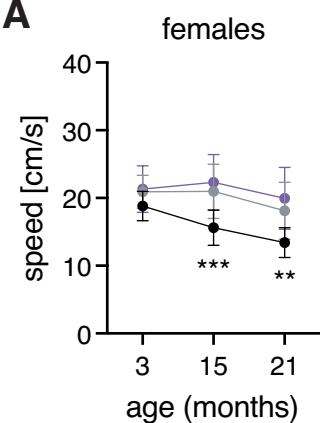

$\rightarrow$ WT $\rightarrow \mathrm{CMV}^{-\mathrm{cre}^{+-}}$

C

$\mathbf{E}$

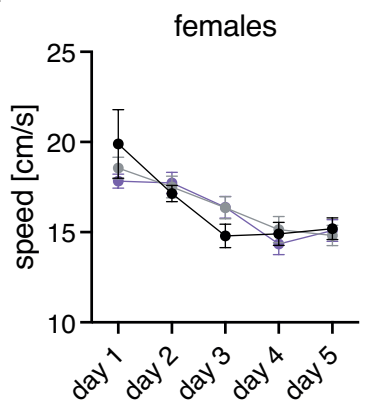

males

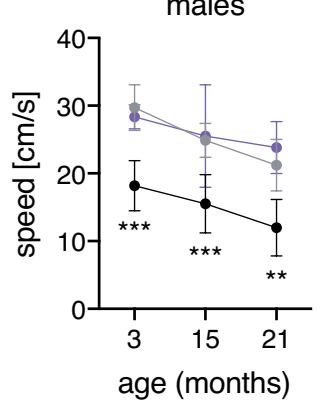

- huGFAT1 wt OE
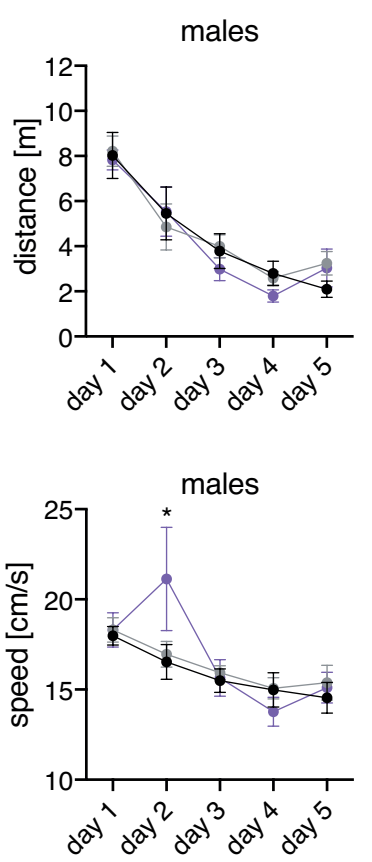

B
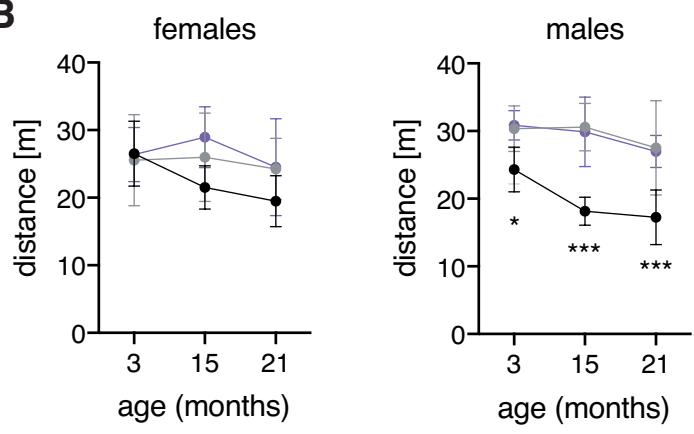

$\rightarrow$ WT $\rightarrow$ CMV-cre ${ }^{+/}$

- huGFAT1 wt OE

D
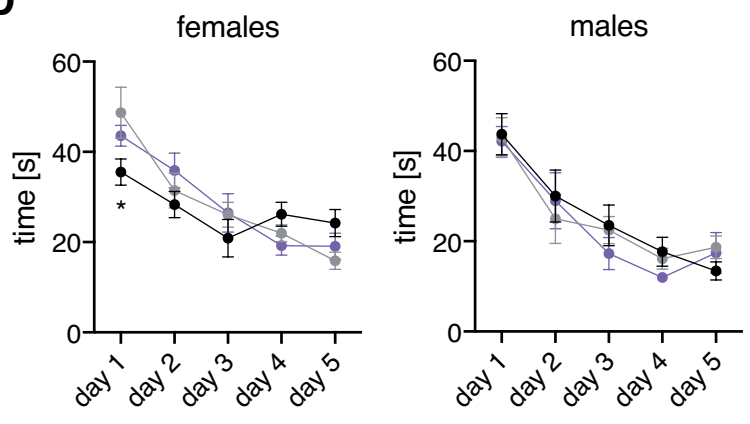

$\rightarrow$ WT

$\rightarrow$ CMV-cre+r-

$\rightarrow$ huGFAT1 wt OE

857 Figure S5: HBP activation by huGFAT1 wt OE does affect behavior or learning
858 of mice. (A) Speed measured in the open field test of control and huGFAT1 wt OE 859 mice of both sexes at 3,15 and 21 months of age. (B) Distance measured in the $860 Y$ maze test of control and huGFAT1 wt OE mice of both sexes at 3, 15 and 21 months 861 of age. (A-B) Data are presented as mean \pm SD $(n \geq 4)$. (C) Distance and (D) Time until 862 the mice reached the hidden platform, and (E) Swimming speed of control and 863 huGFAT1 wt OE mice of both sexes at 4 months of age during the Morris water maze 864 training. (C-E) Data are presented as mean \pm SEM $(n \geq 6)$. The mean of four trials per 865 day is plotted. (A-E) Two-way ANOVA, Dunnett's post-test. Statistical significance was calculated compared to CMV-cre ${ }^{+/}$mice at each time point; only significant changes are indicated. ${ }^{* *} p<0.001 ;{ }^{* *} p<0.01 ;{ }^{*} p<0.05$ 